GEOLOGICAL SURVEY OPEN-FILE REPORT 87-155

\title{
STRONG-MOTION DATA FROM THE JULY 8, 1986 \\ NORTH PALM SPRINGS EARTHQUAKE AND AFTERSHOCKS
}

\author{
RONALD L. PORCELLA \\ EDWIN C. ETHEREDGE \\ RICHARD P. MALEY \\ JOSEPHINE C. SWITZER
}

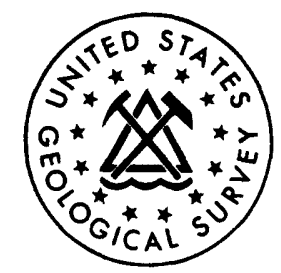

This repoet is preliminary and has not been reviewed for conformity with U.S. Geological Survey editorial standards. 


\section{CONTENTS}

Page

Introduction - - - - - - - - - - - - - - - 1

Main-shock data _. - . . . . . . . . . . . . . . 2

Aftershock data _- $-\ldots \ldots+\ldots$

Acknowledgements _ - $\ldots \ldots \ldots+\ldots$

References - - - - $-\ldots+\ldots$

\section{ILLUSTRATIONS}

Figure 1. USGS accelerograph stations in the southern California region - 3

2. Close-in accelerograph stations - . - . - . - . - 4

3. Selected main-shock records from stations within $135 \mathrm{~km}$ of the

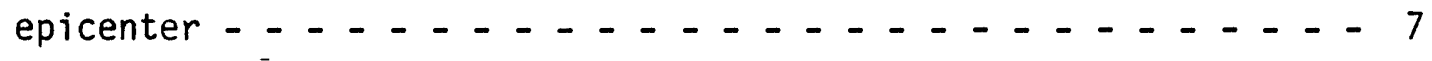

4. Selected aftershock accelerograms _ . . . . . . . - 21

\section{TABLES}

Table 1. Main-shock accelerograph data - $\ldots \ldots$

2. Aftershock accelerograph data $\ldots \ldots$ 


\section{STRONG-MOTION DATA FROM THE JULY 8, 1986 NORTH PALM SPRINGS EARTHQUAKE AND AFTERSWHOCKS}

\section{INTRODUCTION}

A magnitude 5.9 ( $M_{L}$ ) earthquake occurred approximately $15 \mathrm{~km}$ north of Palm Springs, California on July 8, 1986 at 0920:44.0 G.m.t. This event reportedly caused more than $\$ 5$ million in damage, injured 29 people, and destroyed or damaged 51 homes; it was felt throughout much of southern California and was given a maximum MMI rating of VI (USGS, 1986). Preliminary studies located rupture on the Banning fault, with the middle of the hypocentral distribution at an approximate depth of $11 \mathrm{~km}$; surface fractures were located along more than $6 \mathrm{~km}$ of the fault trace immediately west of the town of North Palm Springs (EERI, 1986). The epicenter was located at $33.97^{\circ} \mathrm{N}$ 1at. and $116.61^{\circ} \mathrm{W}$ long.

The main shock triggered accelerographs at more than 45 strong-motion stations operated by the U.S. Geological Survey (USGS) in the southern California region (fig. 1). These stations include 30 ground sites, nine buildings, seven dams, and one freeway interchange. Additionally, accelerographs at more than 30 stations operated by the California Division of Mines and Geology were triggered, including instruments located at 21 ground sites, eight buildings, two dams, and one power plant (OSMS, 1986). Low-level accelerations were recorded at 18 stations in the Los Angeles area operated by the University of Southern California and at one station operated by Kinemetrics, Inc., in Pasadena (EERI, 1986). 
This report contains station-location information, trigger times, and maximum acceleration and strong-duration data for all USGS-operated accelerographs triggered during the July 8 main shock and all aftershocks that occurred through October 15, 1986 (tables 1 and 2).

\section{MAIN-SHOCK DATA}

Table 1 contains station information and record data from 46 USGS strong-motion stations that recorded the July 8 main shock; epicentral distances range between 5 and $180 \mathrm{~km}$.

Nineteen stations, within $50 \mathrm{~km}$ of the epicenter, produced peak horizontal ground accelerations ranging between 0.045 and $0.704 \mathrm{~g}$. Peak vertical ground accelerations of 0.038 to $0.778 \mathrm{~g}$ were recorded at these stations and surpassed corresponding peak horizontal maxima at three of the four closest stations (Morongo Valley, N. Palm Springs, and Cabazon). Strong durations (acceleration greater than $0.10 \mathrm{~g}$ ) exceed 3 seconds at five of the six stations within $30 \mathrm{~km}$ of the epicenter (table 1, fig. 2).

Figure 3 shows copies of all main-shock accelerograms with peak horizontal ground motions greater than $0.05 \mathrm{~g}$ and structure motions greater than $0.10 \mathrm{~g}$; 21 contain good WWVB time codes. Note the contrast in prominent frequencies on the Morongo Valley record with relatively long-period horizontal motion on both the north-south and east-west components, compared to the higher frequencies on the vertical component. Similar response characteristics are not seen on aftershock records or on numerous minor records obtained at this station after previous earthquakes. Relatively high-frequency motions can be seen on main-shock records from the Keenwild, Cranston, and Santa Rosa Mountain stations ( $f i g .3)$. 


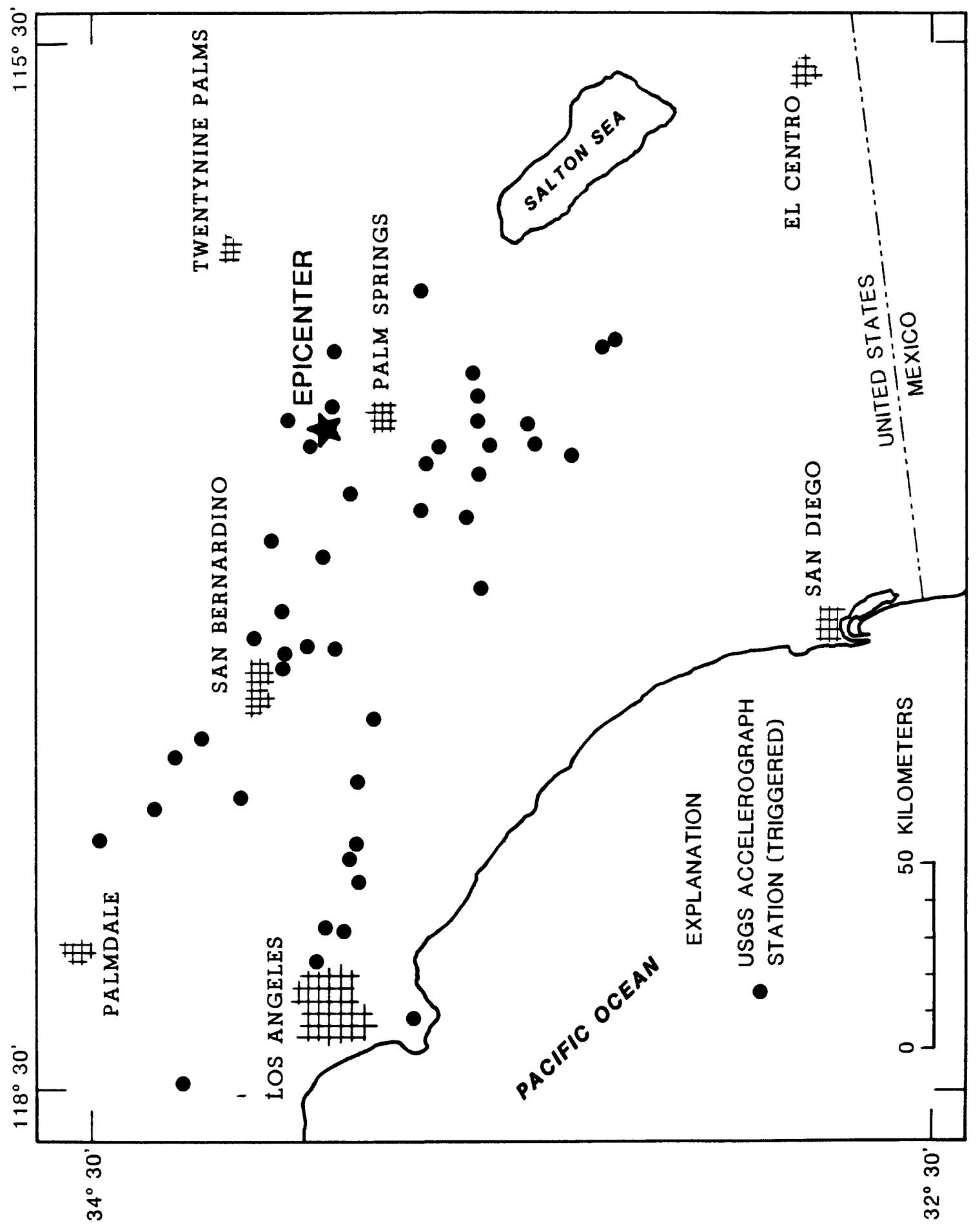

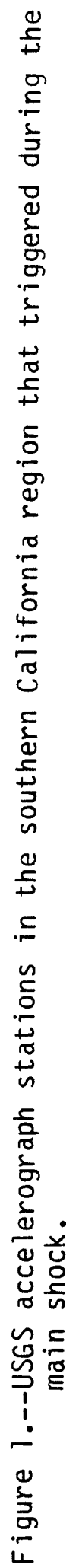




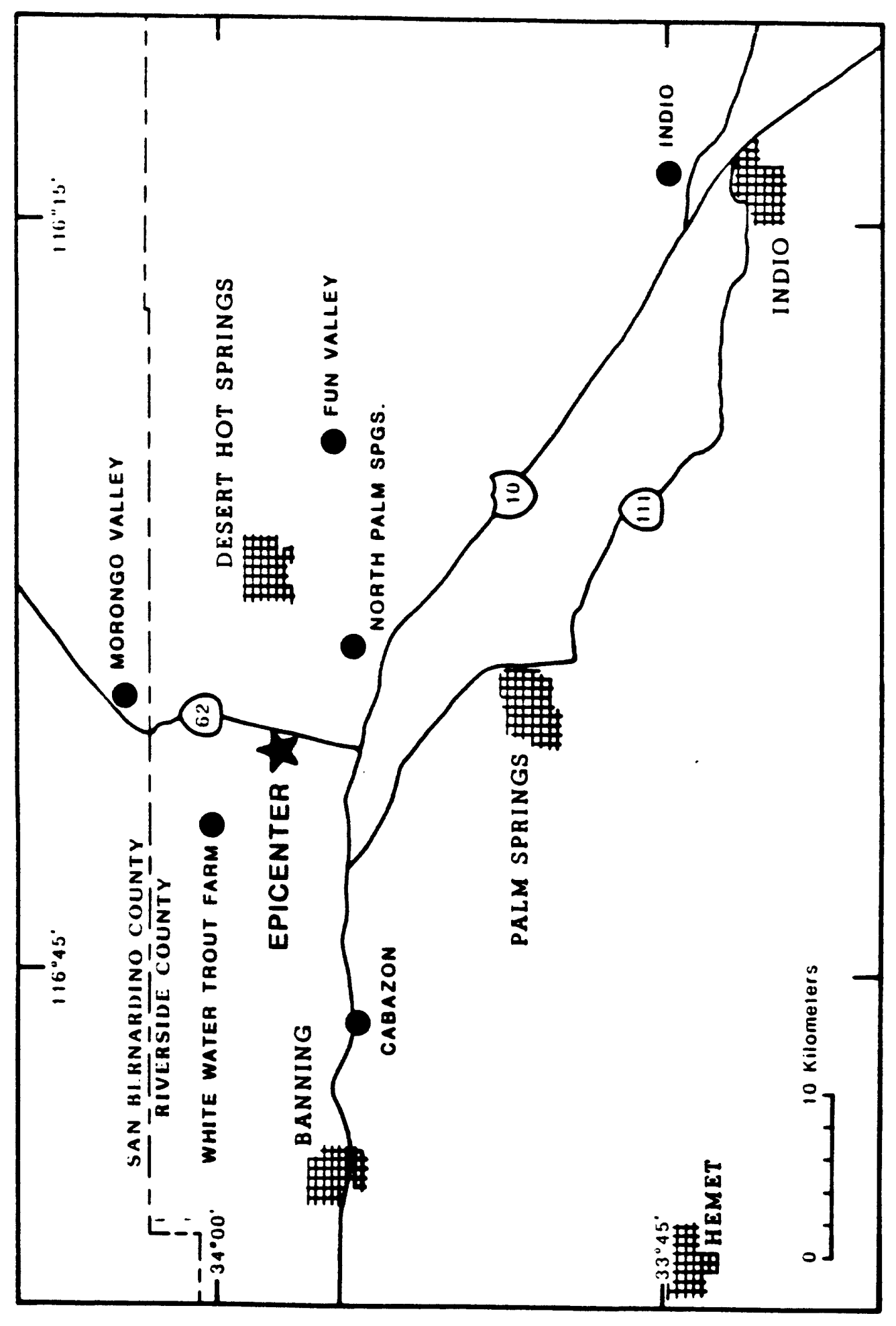

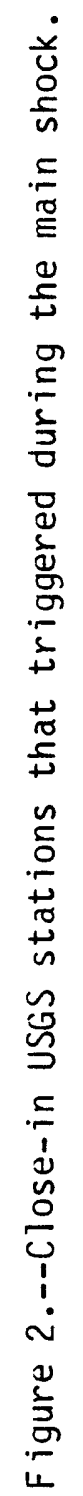




\section{AFTERSHOCK DATA}

Table 2 contains station information and record data from 23 USGS stations that recorded 120 aftershocks during the period July 8 through October 15 (Julian day numbers 189 through 288), 1986. These stations include 4 temporary aftershock stations that were established during the first 24 hours after the main shock, and are designated Desert Hot Springs, Mission Lakes; West Palm Springs Village; Millard Canyon; and Canyon House, Morongo Valley (table 2). Only 82 of these records (68 percent) contain good WWVB radio times, largely due to near-total failure of the radio receiver at the Whitewater Trout Farm station, where 33 aftershocks were recorded. Additional stations where significant numbers of aftershocks were recorded include Morongo Valley (16), North Palm Springs (26), Cabazon (5), and Desert Hot Springs, Mission Lakes (13). Figure 4 contains copies of the 23 aftershock accelerograms that contain peak horizontal ground motions greater than 0.05 g. About 80 percent of the identifiable aftershocks were recorded within one hour of the main shock. The maximum acceleration recorded during any aftershock is $0.168 \mathrm{~g}$ recovered from the North Palm Springs station and is related to the magnitude 4.2 event that occurred approximately 49 minutes after the main shock.

\section{ACKNOWLEDGEMENTS}

The USGS is grateful to the many property owners who have provided space

for the long-term operation of accelerographs, and to those organizations that have cooperated with the USGS in the strong-motion program, including the Metropolitan Water District of Southern California, the Army Corps of Engineers, the Veterans Administration, the California Dept. of 
Transportation and Bechtel Power Corporation. The strong-motion network in this region was maintained by John Nielson and Frank Risavich. Arnie Acosta, Dennis Johnson and Marion Salsman assisted in the collecting and processing of records.

\section{REFERENCES}

U.S. Geological Survey, 1986, Preliminary determination of epicenters: U.S. Department of The Interior, Geological Survey, Washington, D.C. 20402.

E.E.R.I., 1986, Report on the North Palm Springs, California, earthquake July 8, 1986: Special Report, Earthquake Engineering Research Institute, El Cerrito, Calif. $94530,12 \mathrm{p}$.

O.S.M.S., 1986, CSMIP strong-motion records from the Palm Springs, California earthquake of 8 July 1986: Office of Strong-Motion Studies Report OSMS 86-05, $74 \mathrm{p}$. 
- malliftedy

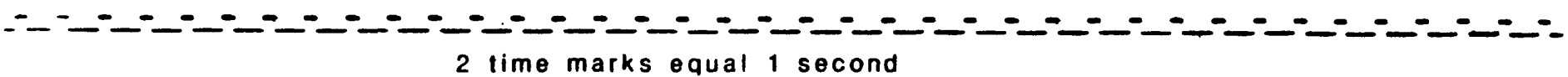

- MORONGO VALLEY

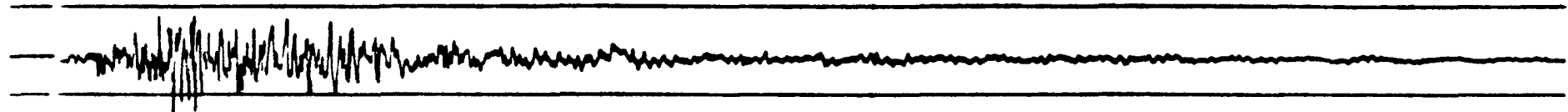

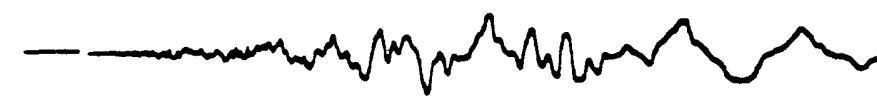

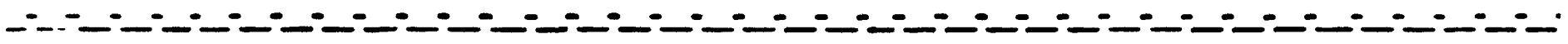
-

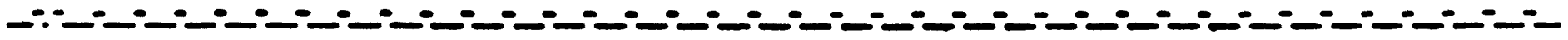
-

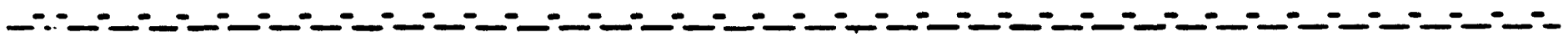

Figure 3.--Main-shock accelerograph records that contain peak ground motions greater than $0.05 \mathrm{~g}$ and peak structure motions greater than $0.10 \mathrm{~g}$. 
r.

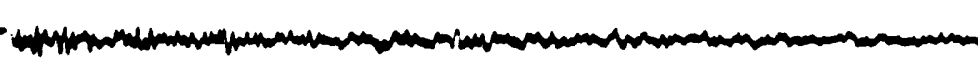

tundumaty

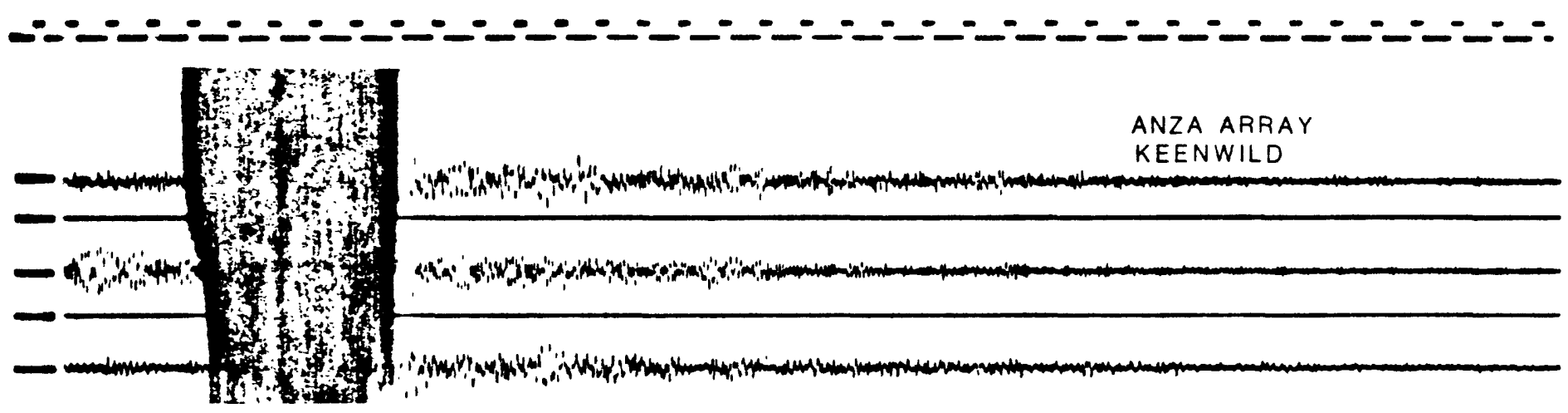

COMMENT: 'Light leak through cover gasket'

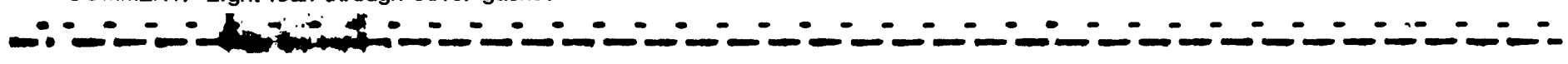

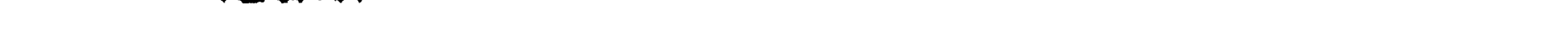

FOREST FALLS

$\therefore-1-1-$

CRANSTON

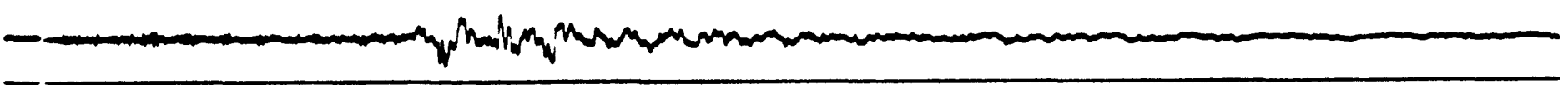

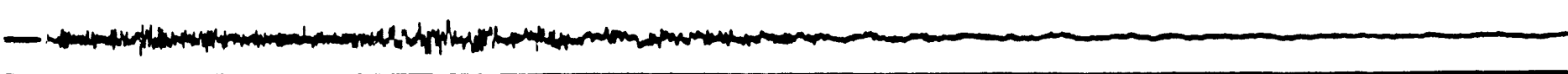

-1 -

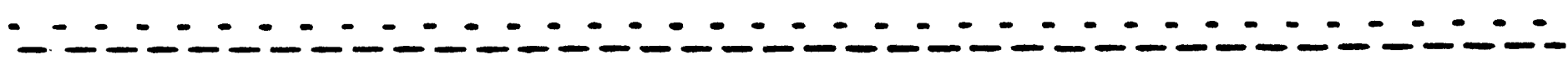

Figure 3.--Continued. 


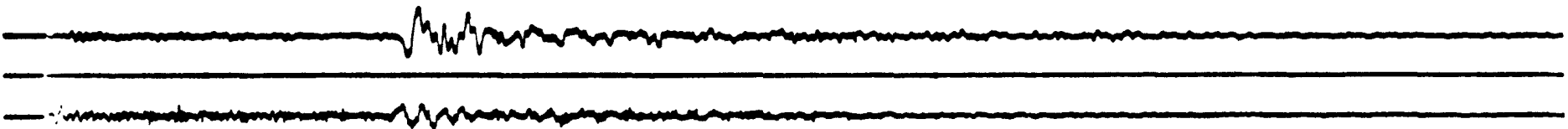
T$\sqrt{M}$ h

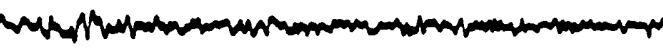

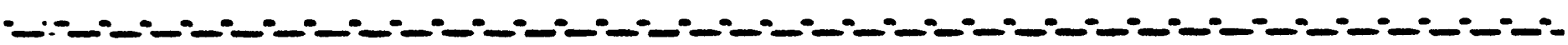

\section{CHERRY VALLEY}

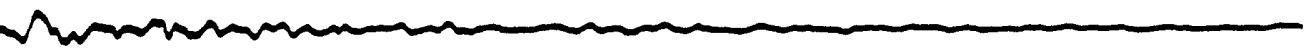

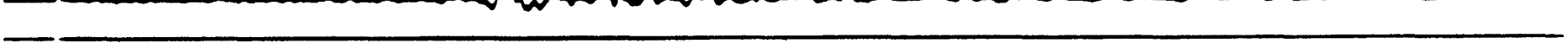

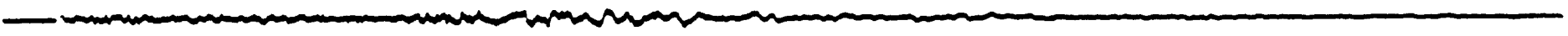
$-1$

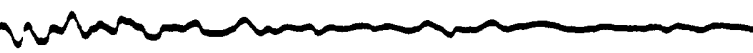

$\therefore-C-$

PINYON FLAT

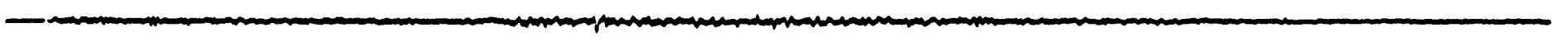

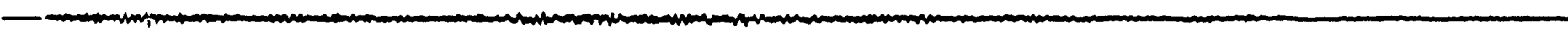

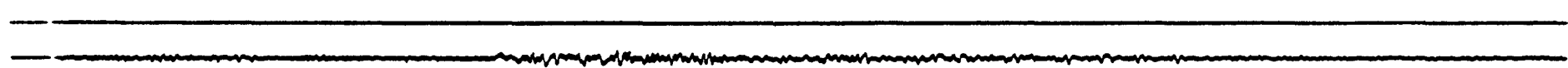

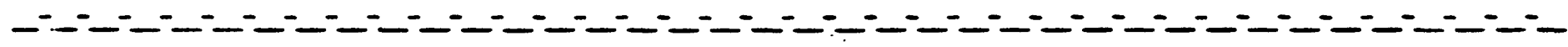

ANZA ARRAY

RED MOUNTAIN

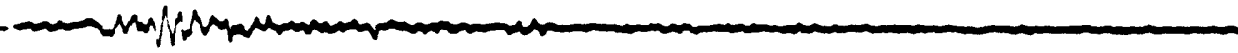

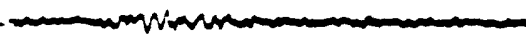

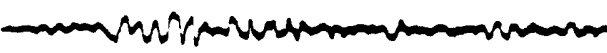

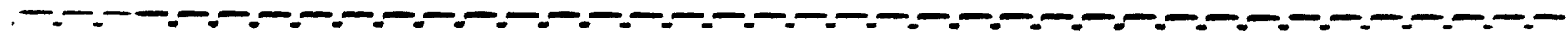

Figure 3.--Continued. 
ANZA ARRAY

TRIPP FLATS

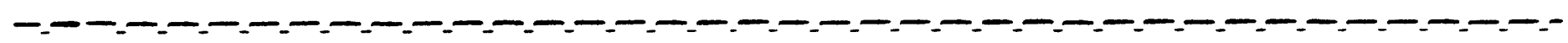

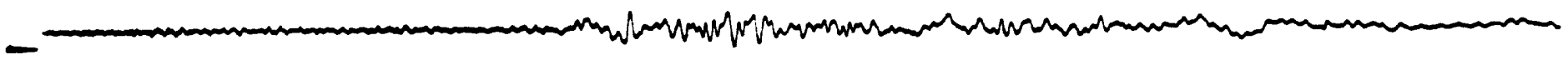

$-$

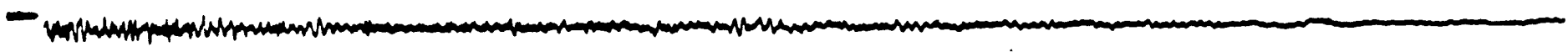

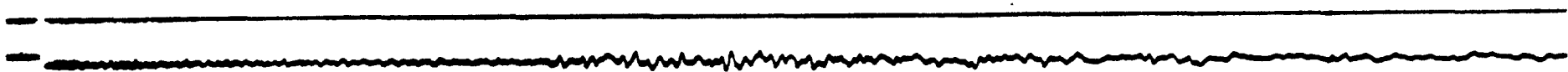

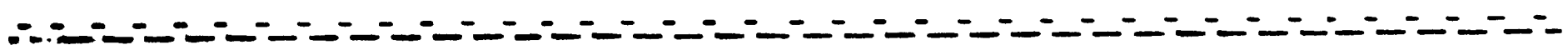
ANZA ARRAY

PINE MEADOWS

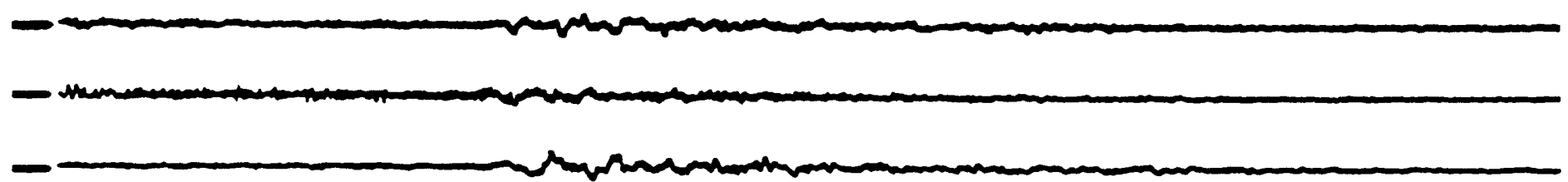

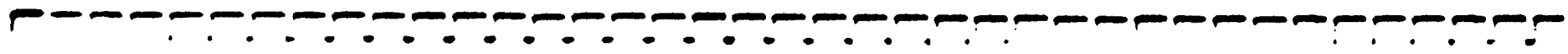

ANZA ARRAY

SANTA ROSA MT.

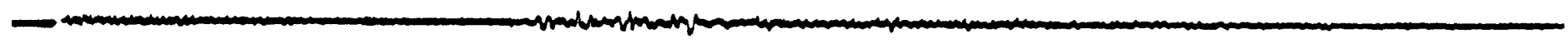

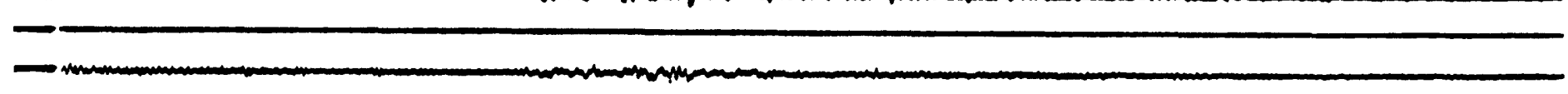

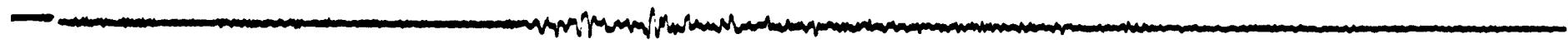

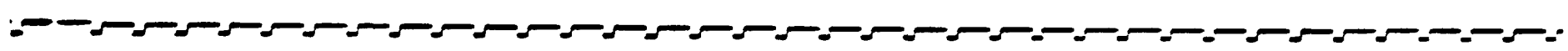

Figure 3.--Continued. 


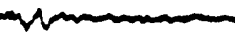

Nommon

$\therefore--$

MENTONE

ר

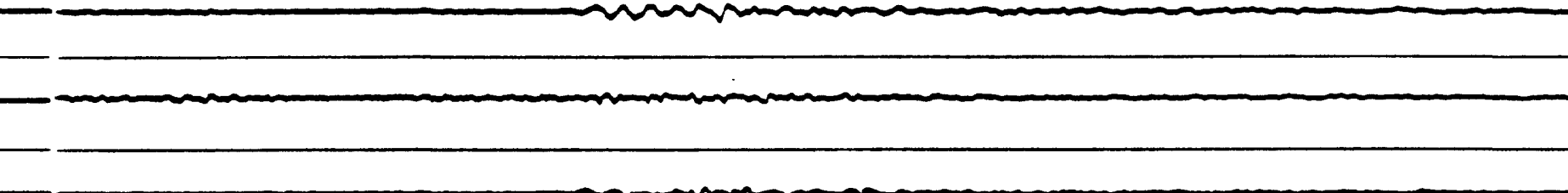

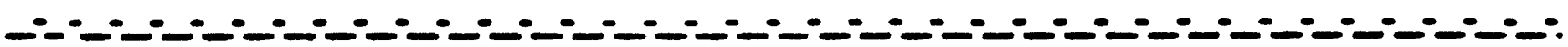

SUNNYMEAD

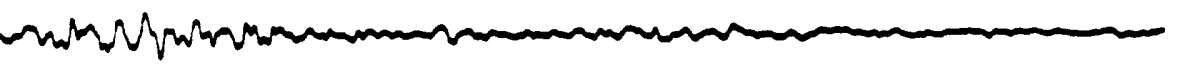

-

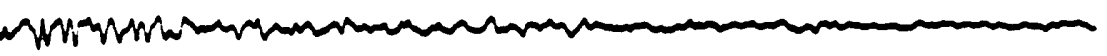

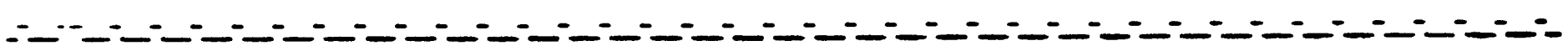

TERWILLIGER VALLEY

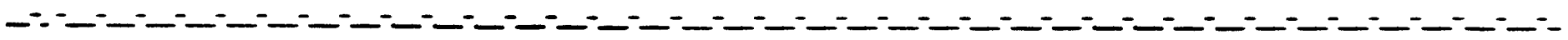

Figure 3.--Continued. 


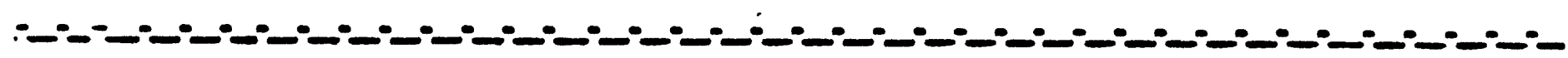
$\longrightarrow$

TULE CANYON

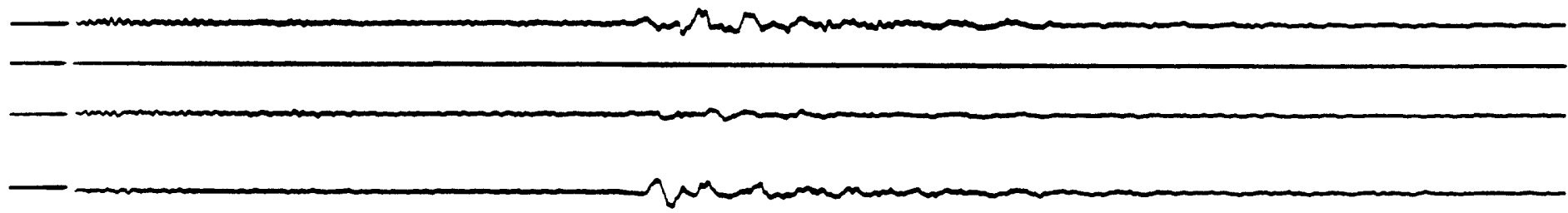
LOMA LINDA VA
SOUTH FF

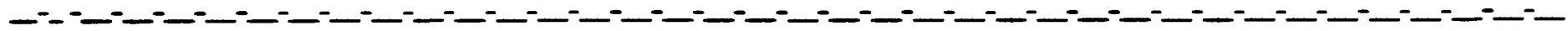

NORTH FF

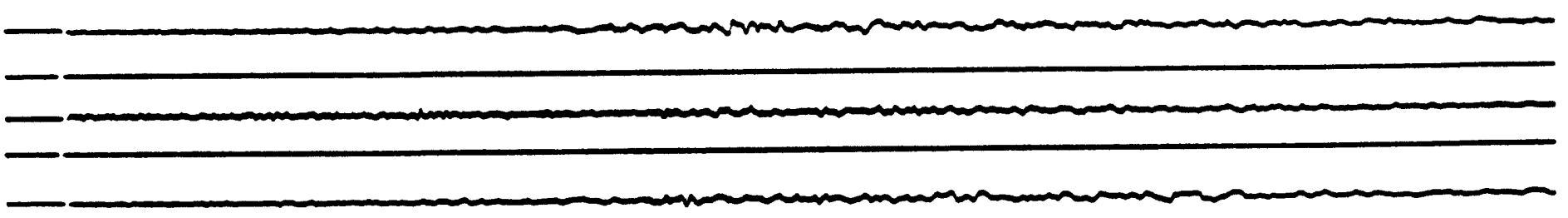

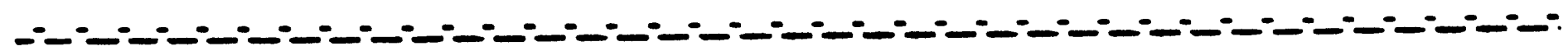

Figure 3.--Continued. 
HIGHLAND

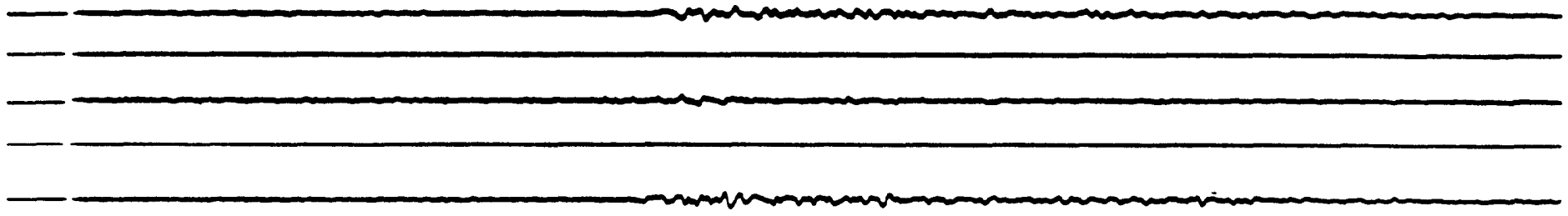

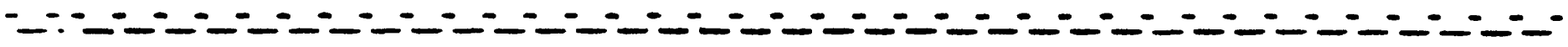

\section{LOMA LINDA UNIV. MEDICAL CENTER}

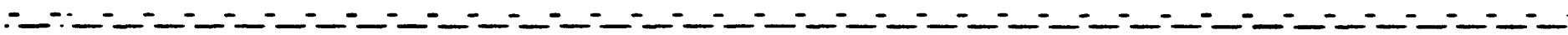

:- - - - - - - - - - - -

LOMA LINDA VA $(9-$ CHANNEL)

$---$

1

2

3

$-\frac{1}{4}$

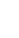

$-$

$-5$

$-$

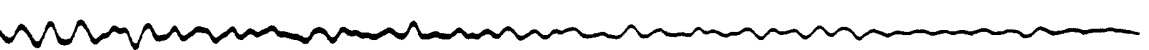

(1)

-

Figure 3.--Continued. 


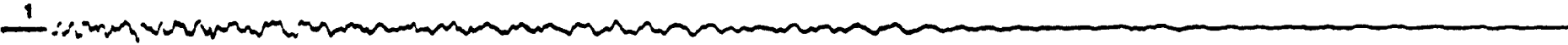$$
2
$$$$
2
$$

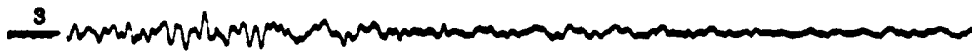

4

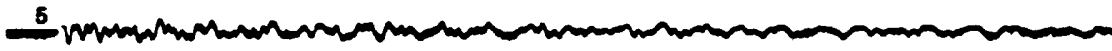

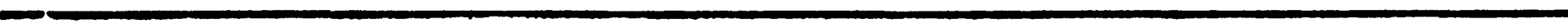

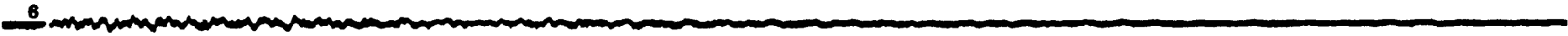

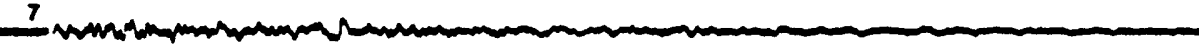

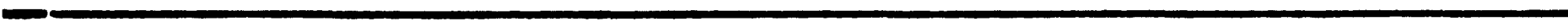

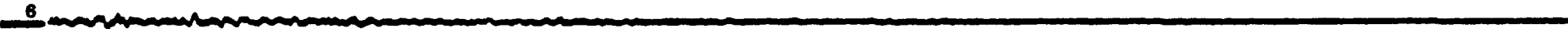

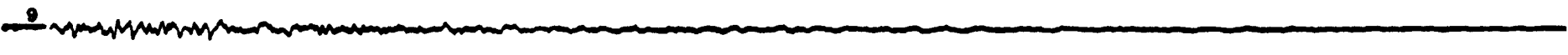

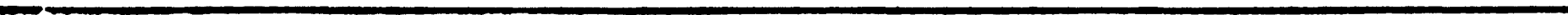

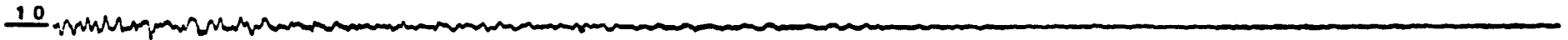

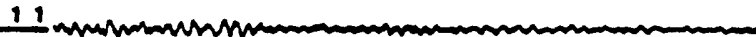

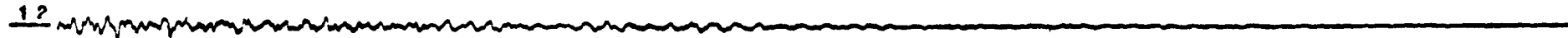

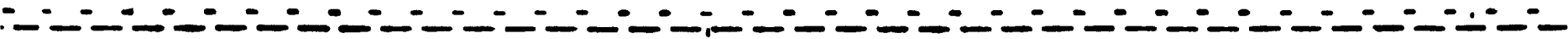

Figure 3.---Continued. 


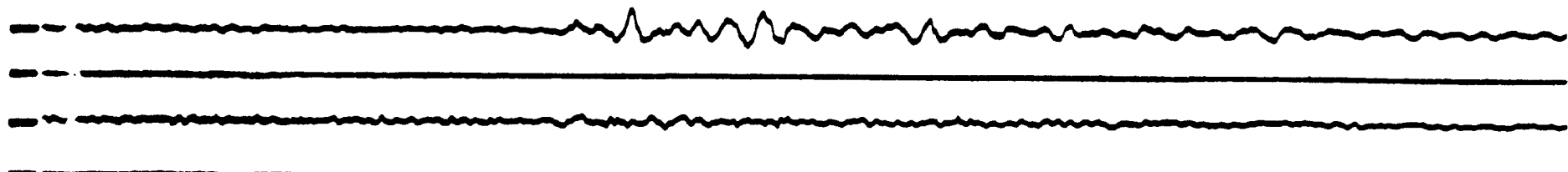

Non

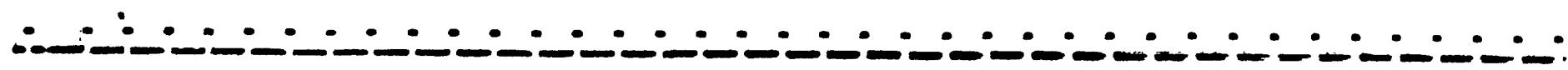

VAULT

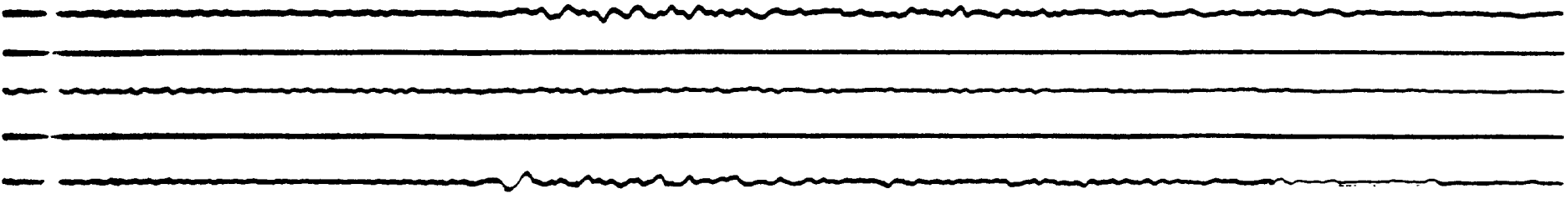

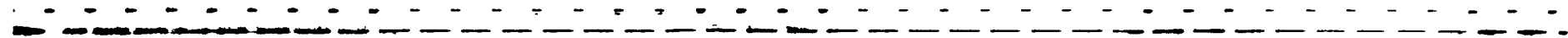
ANZA ARRAY
ANAHUA

CHIHUAHUA VALLEY

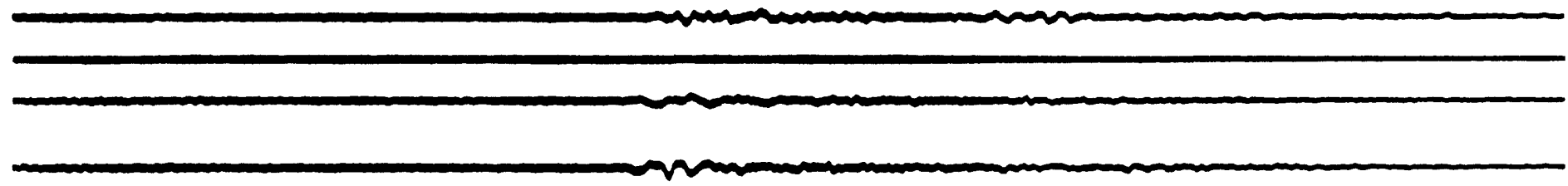

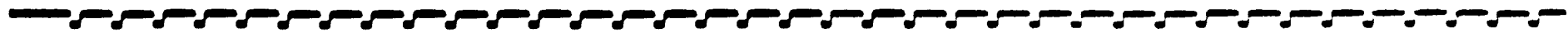

RANCHO DE ANZA

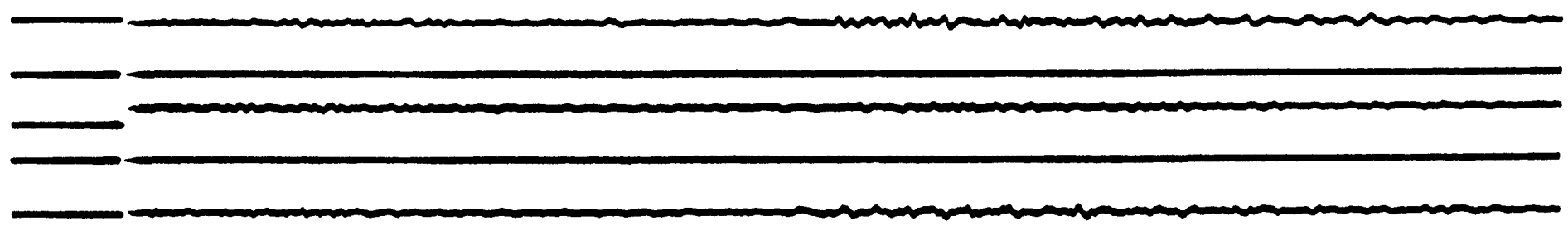

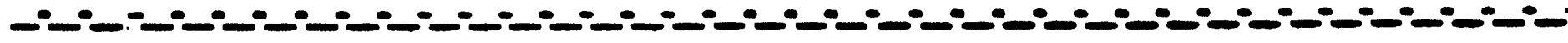

Figure 3.--Continued. 


\title{
LAKE MATHEWS TOE
}

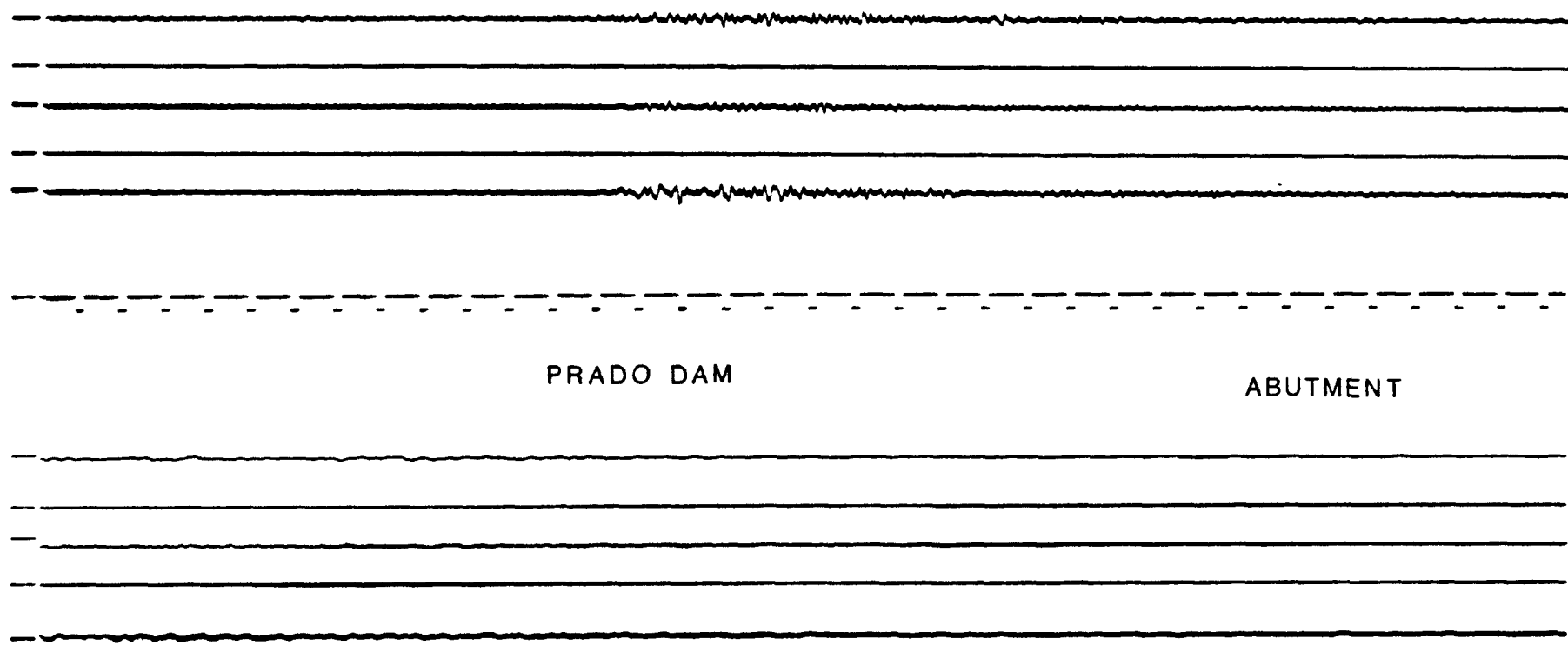

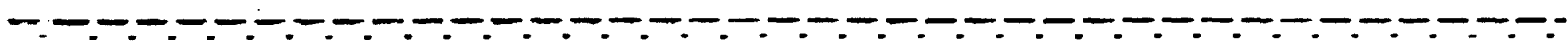

DOWNSTREAM

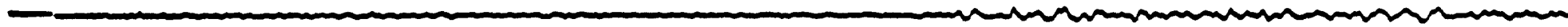

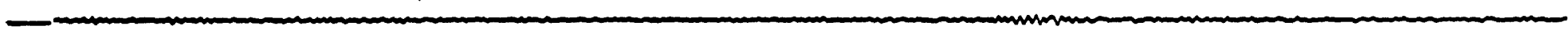

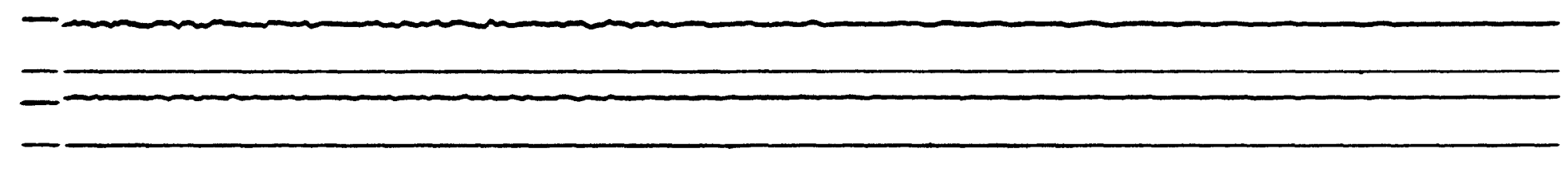




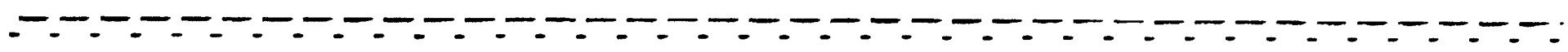
NORWALK 12400 IMPERIAL HIGHWAY
BASEMENT

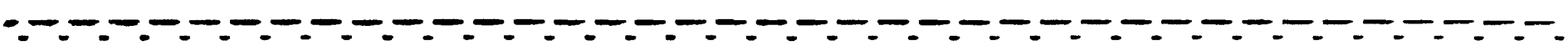

4 in FLOOR

น น

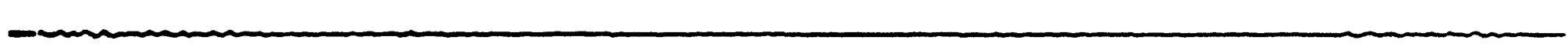
$-$

$-$

ח

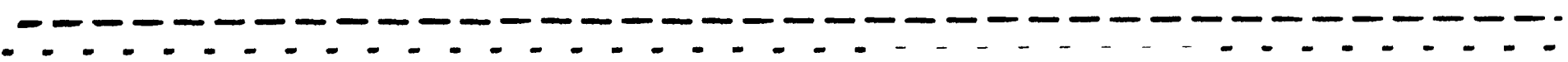

8 th FLOOR

mman

$-$

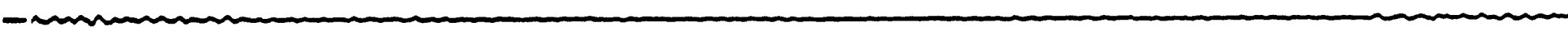

$\sim \sim n$

NORTH FREEFIELD

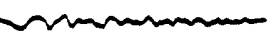

$$
-
$$

$-$

$-$

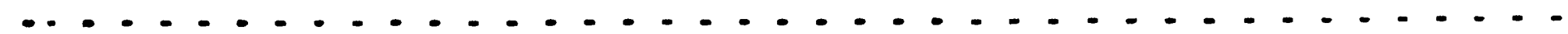

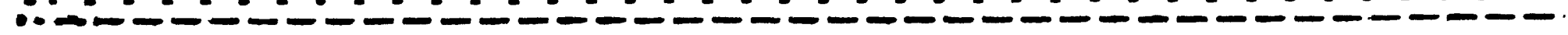

Figure 3.---Continued. 


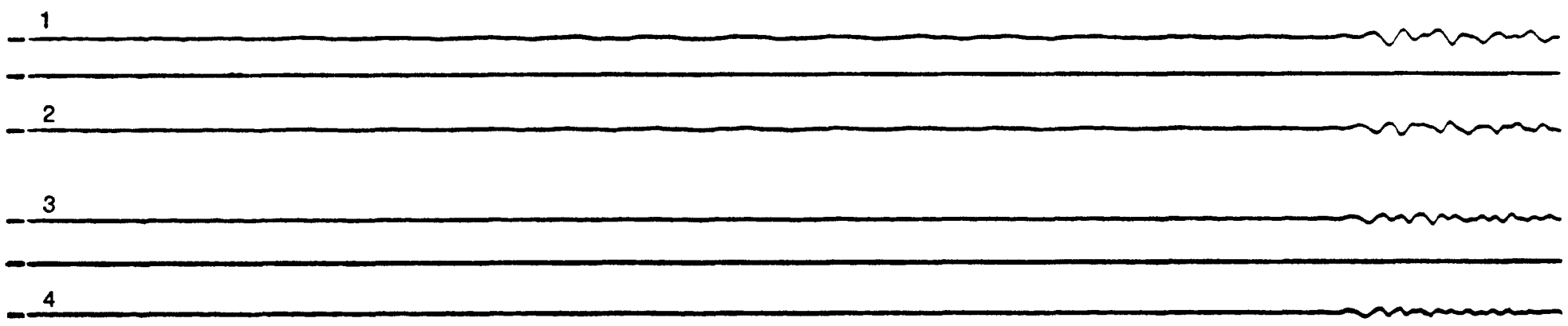

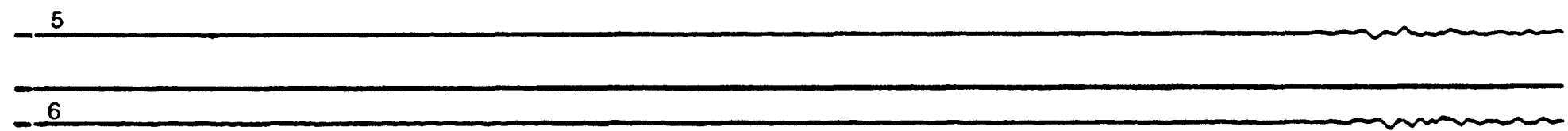

17

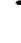

$-8$

$-$

$-10$

$-11$

$-1$

Figure 3.--Continued. 


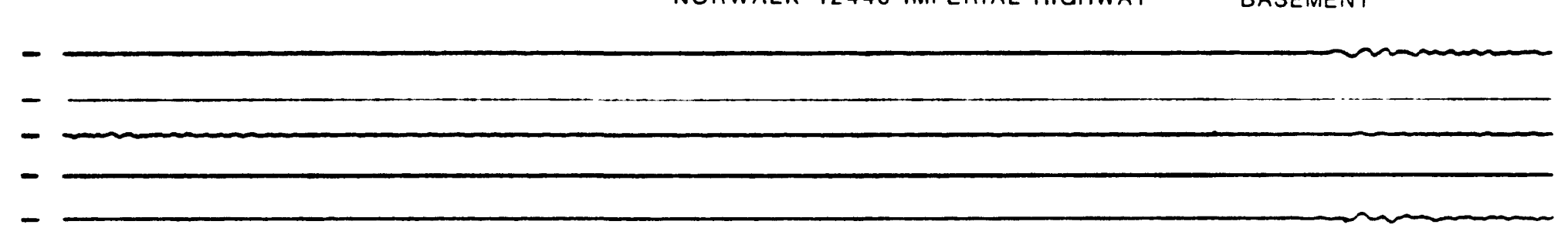

NORWALK 12440 IMPERIAL HIGHWAY GROUND STATION - NORTH

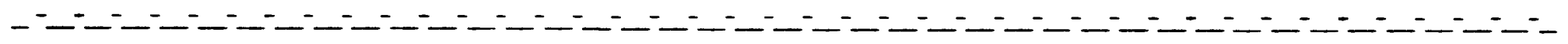

Figure 3.--Continued. 
$-1$

- minimumentivin

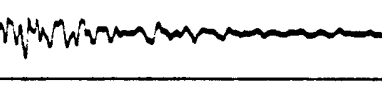

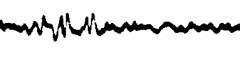

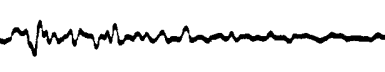

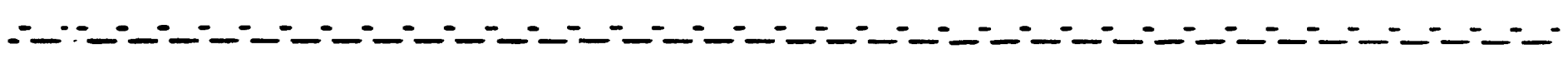

WHITEWATER $7 / 17 / 86 \quad 21: 54$

- -

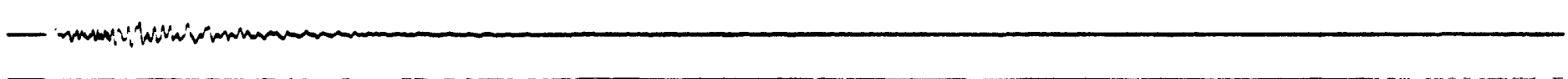

-

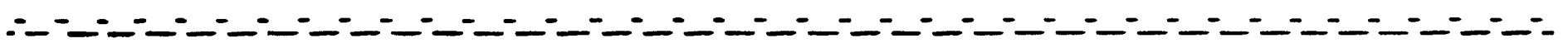

MORONGO VALLEY $7 / 8 / 86 \quad 09: 24$

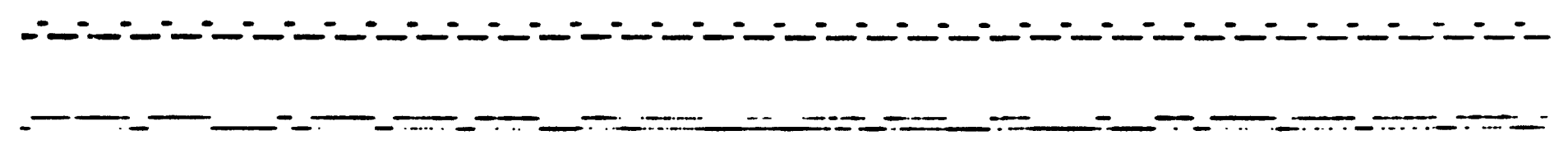

MORONGO VALLEY 7/17/8620:35

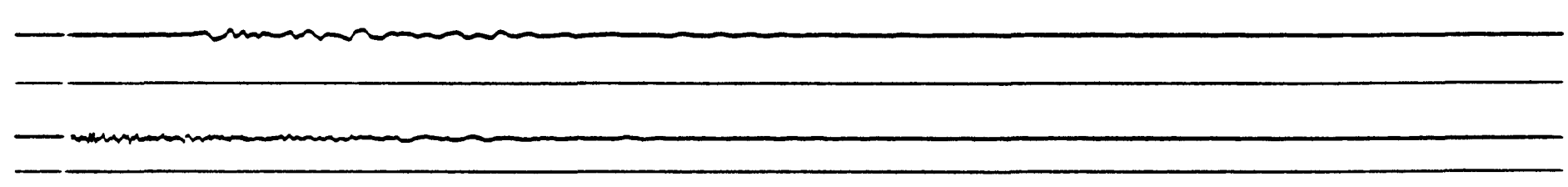

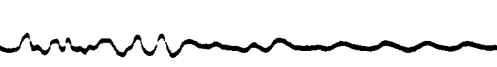

- - - - - - - - - - - - - - - - - - - - - - - - -

Figure 4.--Aftershock accelerograms from the Palm Springs region that contain peak ground motions greater than $0.05 \mathrm{~g}$. 


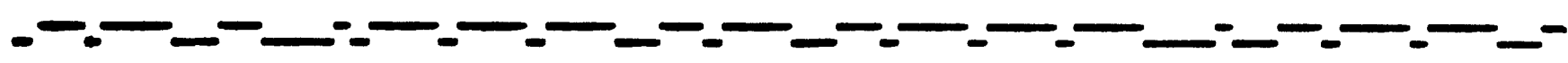

$$
\text { NORTH PALM SPRINGS } \quad 7 / 8 / 86 \quad 09: 24
$$

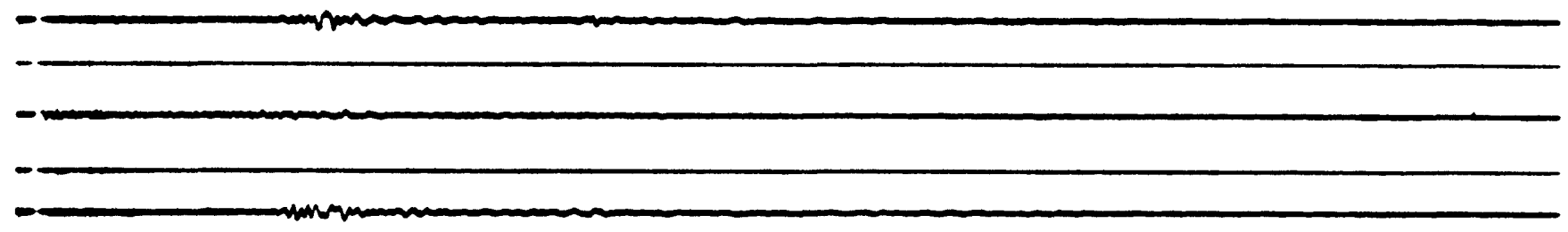

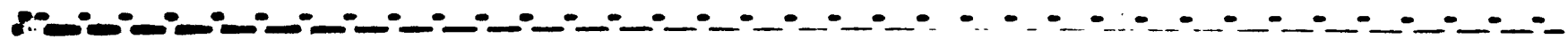
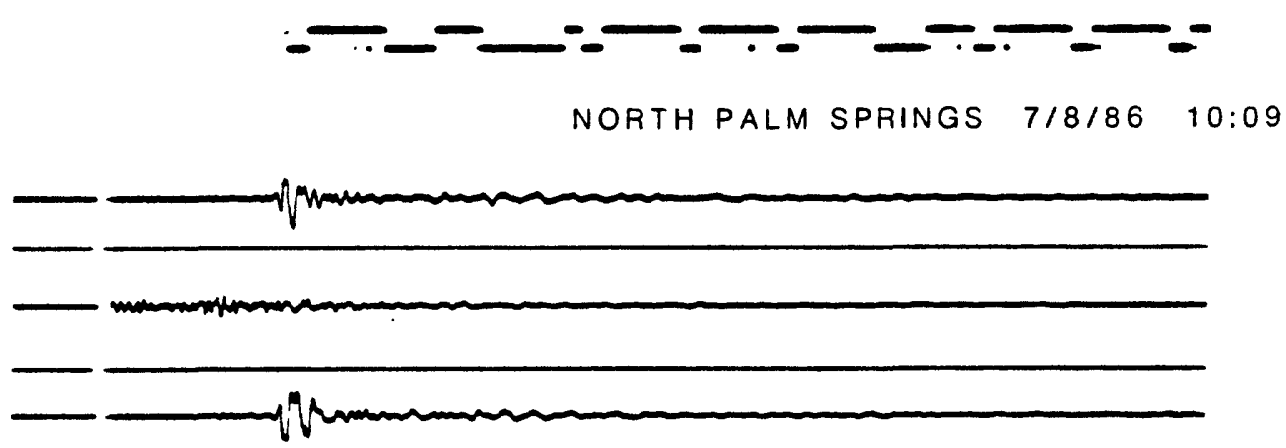

$-$

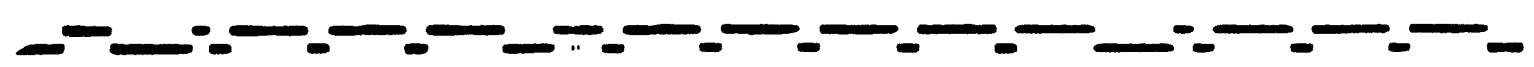

$$
\text { NORTH PALM SPRINGS } \quad 7 / 8 / 86 \quad 10: 11
$$

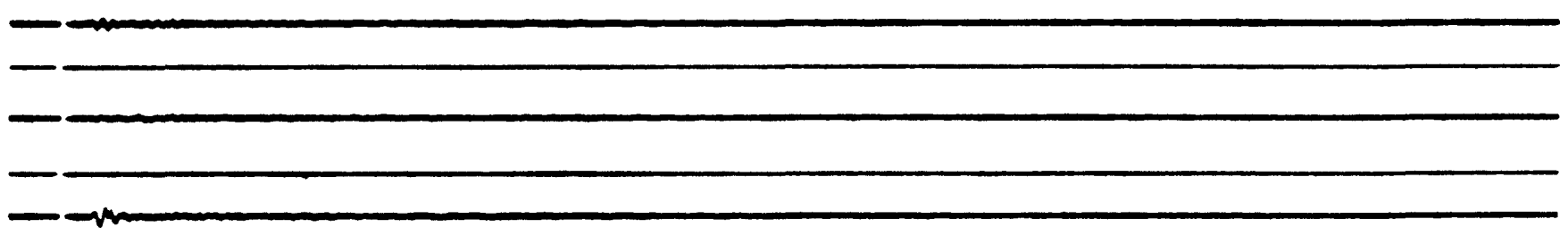

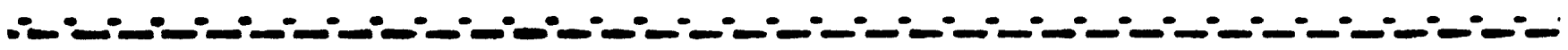

Figure 4.--Continued. 


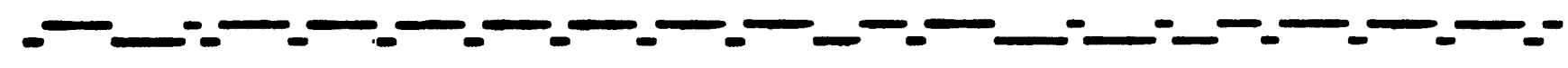

$$
\text { NORTH PALM SPRINGS } 7 / 8 / 86 \quad 16: 39
$$

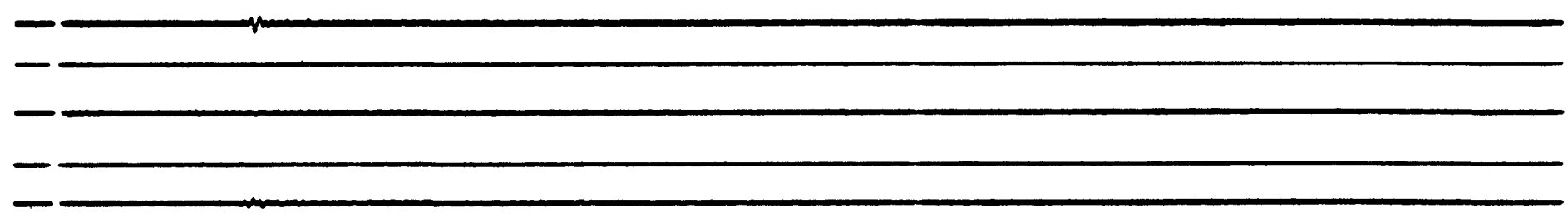

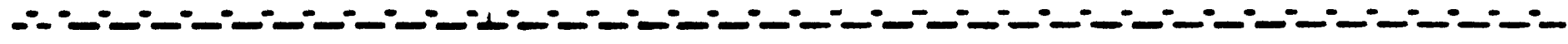

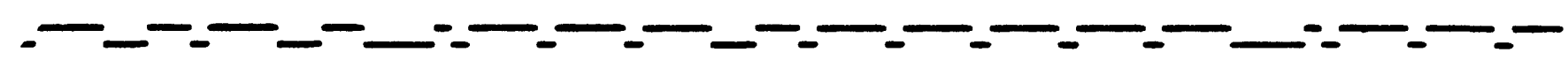

NORTH PALM SPRINGS $7 / 9 / 86 \quad 00: 12$

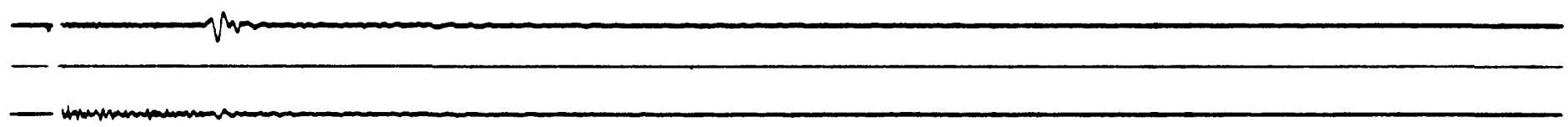

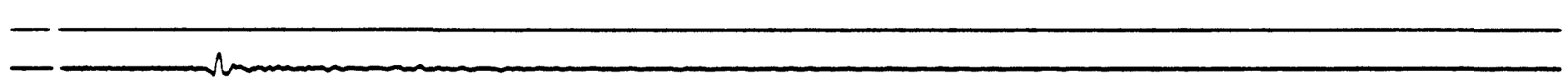

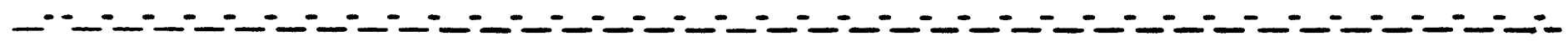

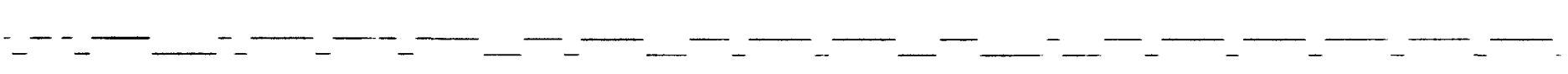

$$
\text { NORTH PALM SPRINGS } 7 / 17 / 86 \quad 20: 35
$$

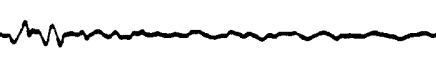

Figure 4.---Continued. 


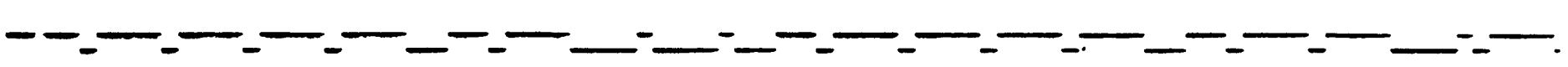

NORTH PALM SPRINGS $7 / 29 / 86 \quad 06: 43$

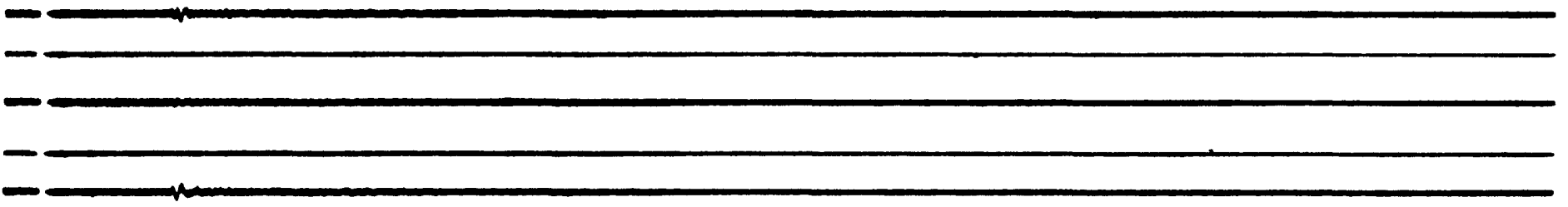

NORTH PALM SPRINGS $8 / 29 / 86 \quad 07: 46$

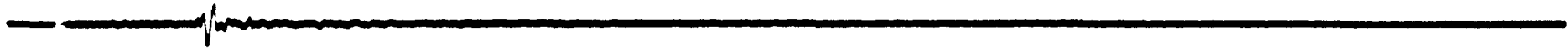

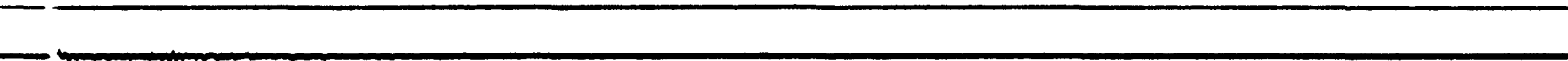

- — ב

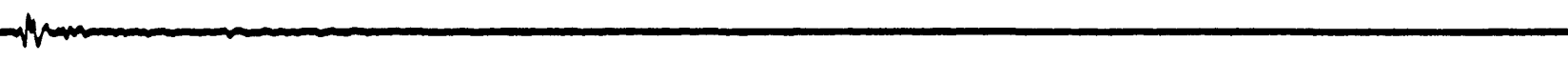

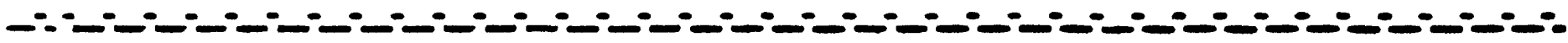

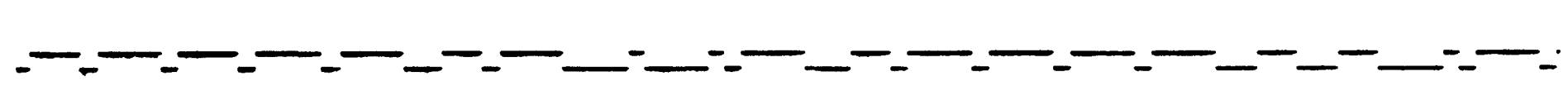

$$
\text { NORTH PALM SPRINGS } 9 / 9 / 86 \quad 16: 22
$$

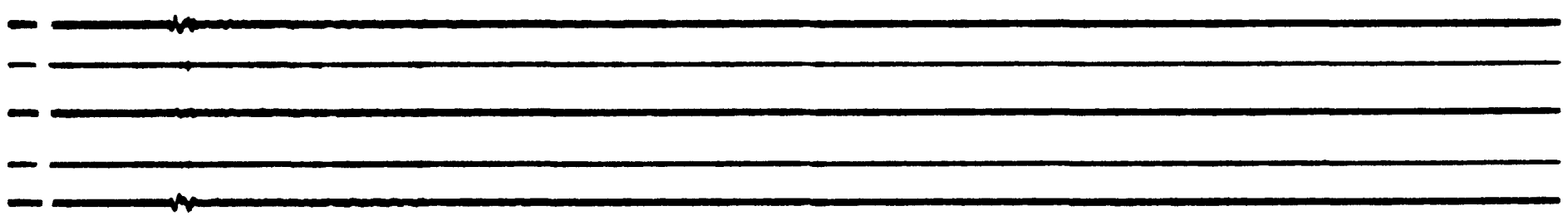

NORTH PALM SPRINGS 10/15/86 $02: 28$

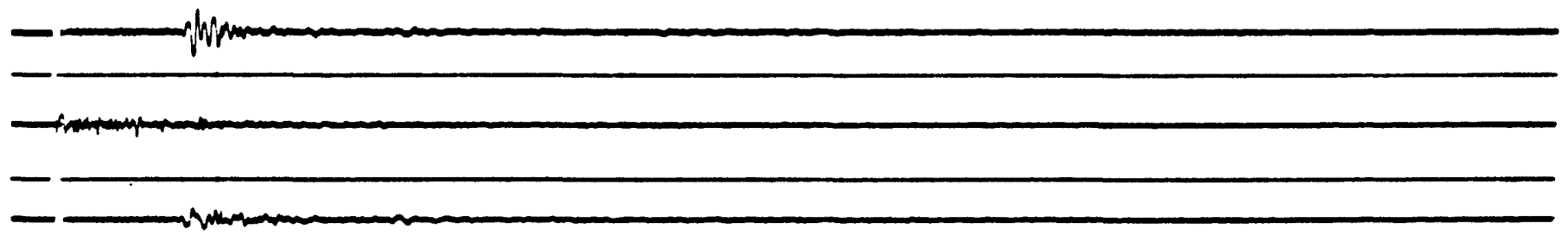

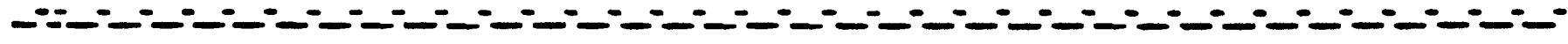

Figure 4.---Continued. 
FUN VALLEY $7 / 8 / 86 \quad 09: 30$

MISSION LAKES $7 / 9 / 86 \quad 00: 12$

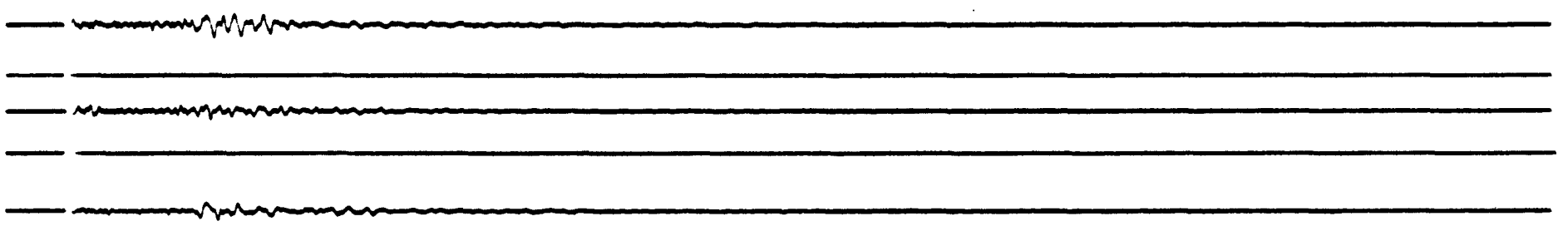

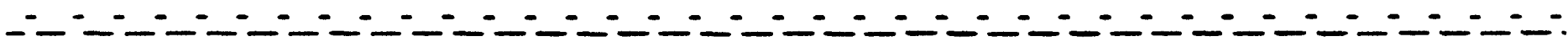

MISSION LAKES $7 / 17 / 86 \quad 20: 35$



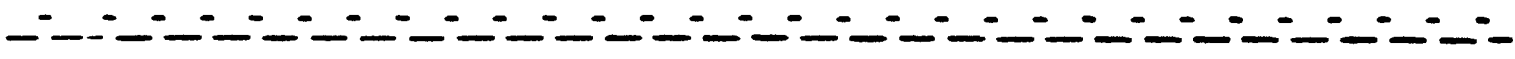

Figure 4.---Continued. 


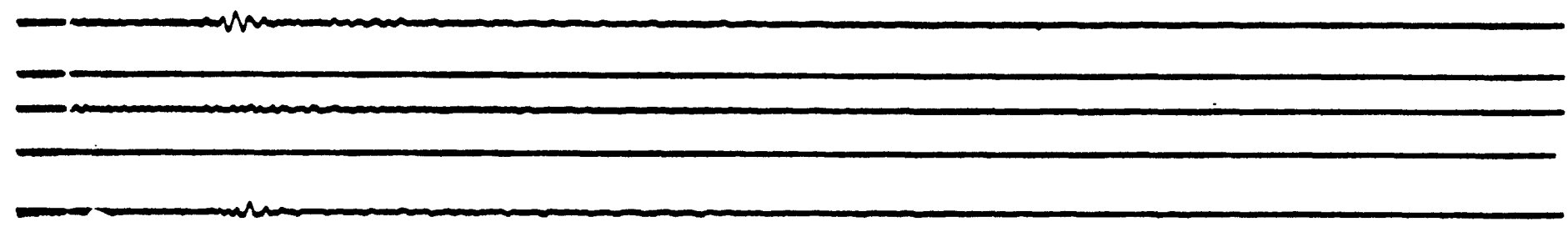

MISSION LAKES $8 / 29 / 86 \quad 07: 46$

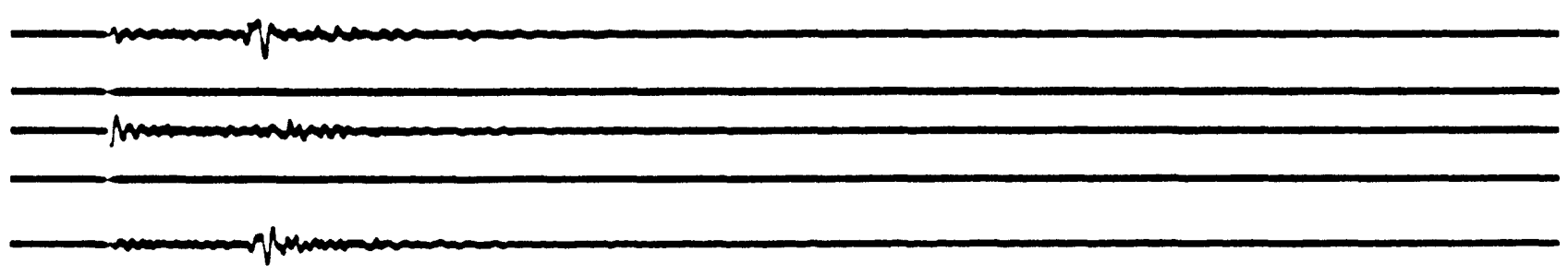

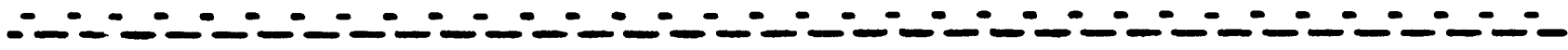

MISSION LAKES $\quad 10 / 15 / 86 \quad 02: 28$

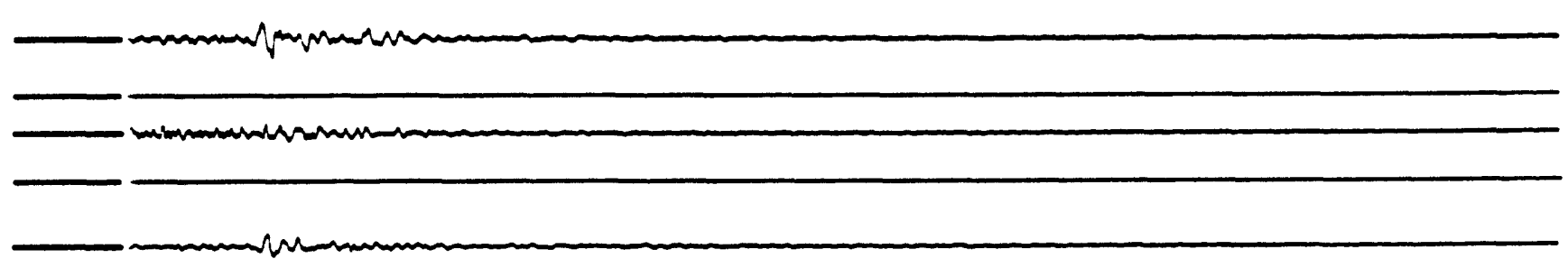

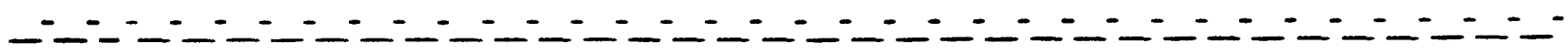

Figure 4.---Continued. 


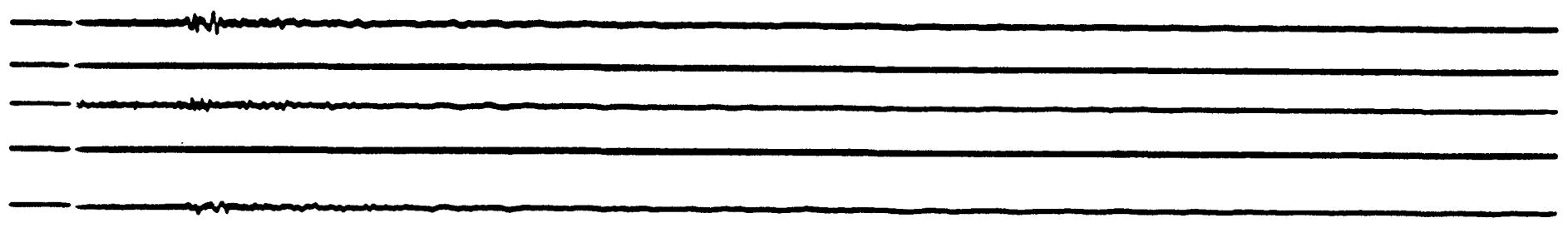

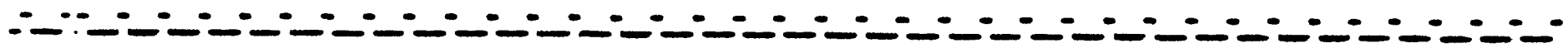

WEST PALM SPRINGS $7 / 17 / 86 \quad 21: 54$

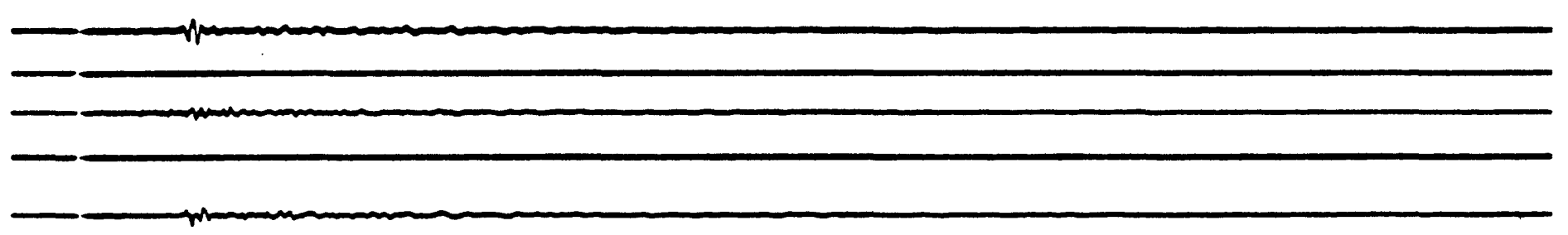

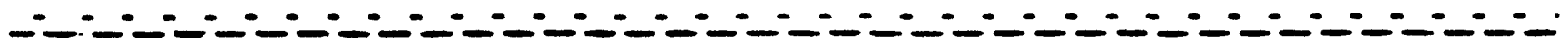

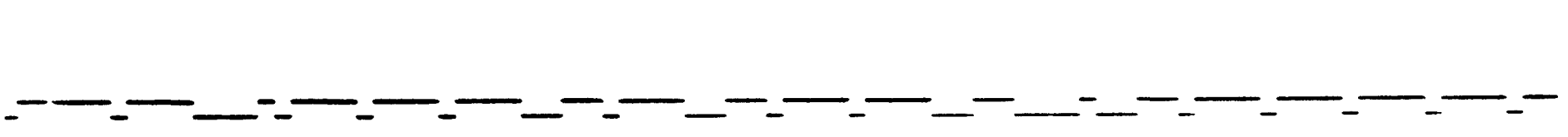

$$
\text { MORONGO, CANYON HOUSE } 7 / 17 / 86 \quad 20: 35
$$

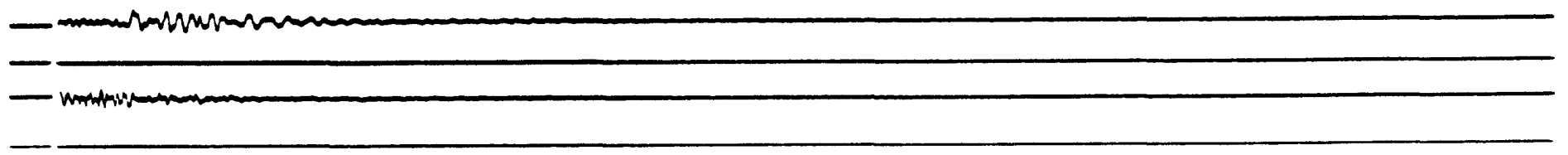

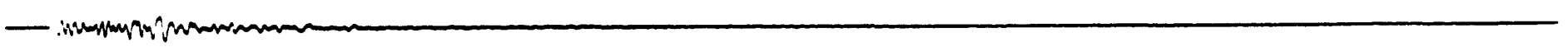

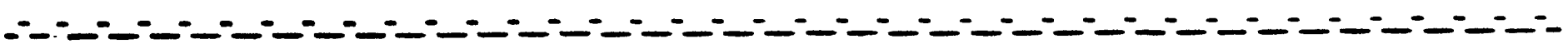

Figure 4.--Continued. 
Table 1.--Main-shock accelerograph data for USGS stations in the greater Palm Springs region

[Epicentral distance and Azimuth are measured relative to epicenter at lat. $33.97^{\circ} \mathrm{N}$., long. $116.61^{\circ} \mathrm{W}$. Trigger times include day (July 8 )/hour:minute:second. S-t interval is time between instrument triggering and arrival of S-wave motion; dash indicates interval is indeterminable. Direction of acceleration is for upward trace deflection on accelerogram; vertical-component directions are listed as "up" or "down". Duration of acceleration is time between first and last peaks of acceleration greater than $0.10 \mathrm{~g}$.

\begin{tabular}{|c|c|c|c|c|c|c|c|c|c|c|}
\hline \multicolumn{4}{|c|}{ Station identification } & \multirow{2}{*}{$\begin{array}{c}\text { Epicentral } \\
\text { distance } \\
(\mathrm{km})\end{array}$} & \multirow{2}{*}{$\begin{array}{l}\text { Azimuth } \\
\text { (degrees) }\end{array}$} & \multirow{2}{*}{$\begin{array}{l}\text { Trigger } \\
\text { time } \\
\text { (G.m.t.) }\end{array}$} & \multirow{2}{*}{$\begin{array}{c}S-t \\
\text { interval } \\
(s)\end{array}$} & \multicolumn{3}{|c|}{ Acceleration } \\
\hline Number & Name & $\begin{array}{l}\text { Coorc } \\
\text { (lat. }{ }^{\circ} \text { N., }\end{array}$ & $\begin{array}{l}\text { dinates } \\
\left.\text { long. }{ }^{\circ} \mathrm{W} .\right)\end{array}$ & & & & & $\begin{array}{l}\text { Direction } \\
\text { (degrees) }\end{array}$ & $\begin{array}{l}\text { Maximum } \\
(\mathrm{g})\end{array}$ & $\begin{array}{l}\text { Duration } \\
\text { (s) }\end{array}$ \\
\hline 5072 & $\begin{array}{l}\text { Whitewater } \\
\text { Trout Farm }\end{array}$ & 33.989 , & 116.655 & 5 & 296 & unknown & 1.6 & $\begin{array}{l}270 \\
\text { Up } \\
180\end{array}$ & $\begin{array}{l}.662 \\
.438 \\
.497\end{array}$ & $\begin{array}{l}4.5 \\
4.9 \\
4.5\end{array}$ \\
\hline 5071 & Morongo Valley & 34.048, & 116.577 & 9 & 017 & $189 / 09: 20: 47.0$ & 1.9 & $\begin{array}{l}135 \\
\text { Up - } \\
045\end{array}$ & $\begin{array}{l}.217 \\
.348 \\
.229\end{array}$ & $\begin{array}{l}4.7 \\
4.2 \\
4.8\end{array}$ \\
\hline 5070 & $\begin{array}{l}\text { N. Palm Springs } \\
\text { Post Office }\end{array}$ & 33.924 , & 116.543 & 9 & 131 & $189 / 09: 20: 47.55$ & 2.0 & $\begin{array}{l}300 \\
\text { Up } \\
210\end{array}$ & $\begin{array}{l}.681 \\
.778 \\
.704\end{array}$ & $\begin{array}{l}6.0 \\
5.6 \\
5.3\end{array}$ \\
\hline 5073 & Cabazon & 33.918 , & 116.782 & 17 & 251 & $189 / 09: 20: 48.50$ & 2.4 & $\begin{array}{l}270 \\
\text { Up } \\
180\end{array}$ & $\begin{array}{l}.209 \\
.376 \\
.223\end{array}$ & $\begin{array}{l}3.3 \\
3.2 \\
2.7\end{array}$ \\
\hline 5069 & Fun Valley & 33.925 , & 116.389 & 21 & 102 & $189 / 09: 20: 48.95$ & 2.8 & $\begin{array}{l}135 \\
\text { Up } \\
045\end{array}$ & $\begin{array}{l}.139 \\
.085 \\
.128\end{array}$ & $\begin{array}{c}0.6 \\
- \\
0.6\end{array}$ \\
\hline 5232 & Keenwild & 33.71, & 116.71 & 30 & 198 & $189 / 09: 20: 50.85$ & 3.9 & $\begin{array}{l}180 \\
\text { Up } \\
090\end{array}$ & $\begin{array}{l}.327 \\
.186 \\
.211\end{array}$ & $\begin{array}{l}4.7 \\
8.1 \\
2.9\end{array}$ \\
\hline 5075 & $\begin{array}{l}\text { Forest Falls } \\
\text { Post Office }\end{array}$ & 34.09 , & 116.92 & 32 & 295 & 189/09:20:50 & 4.0 & $\begin{array}{l}300 \\
\text { Up } \\
210\end{array}$ & $\begin{array}{l}.067 \\
.055 \\
.077\end{array}$ & $\overline{-}$ \\
\hline 5157 & $\begin{array}{l}\text { Cranston } \\
\text { Forest Station }\end{array}$ & 33.74, & 116.84 & 33 & 220 & $189 / 09: 20: 51.40$ & 4.55 & $\begin{array}{l}315 \\
\text { Up } \\
225\end{array}$ & $\begin{array}{l}.188 \\
.126 \\
.139\end{array}$ & $\begin{array}{l}1.5 \\
0.4 \\
1.7\end{array}$ \\
\hline 5043 & Hurkey Creek Park & 33.67, & 116.68 & 34 & 191 & $189 / 09: 20: 51.34$ & 4.30 & $\begin{array}{l}135 \\
\text { Up } \\
045\end{array}$ & $\begin{array}{l}.185 \\
.084 \\
.242\end{array}$ & $\begin{array}{l}1.0 \\
- \\
0.3\end{array}$ \\
\hline $5219^{\circ}$ & Cherry Valley & 33.98, & 116.99 & 35 & 272 & $189 / 09: 20: 51.48$ & 5.2 & $\begin{array}{l}295 \\
\text { Up } \\
205\end{array}$ & $\begin{array}{l}.103 \\
.058 \\
.098\end{array}$ & $\begin{array}{l}1 \text { peak } \\
1 \text { peak }\end{array}$ \\
\hline 5044 & $\begin{array}{l}\text { Pinyon Flat } \\
\text { Observatory }\end{array}$ & 33.61, & 116.46 & 42 & 161 & 189/09:20:52 & 5.4 & $\begin{array}{l}135 \\
\text { Up } \\
045\end{array}$ & $\begin{array}{l}.067 \\
.058 \\
.053\end{array}$ & $\overline{-}$ \\
\hline 5224 & Red Mountain & 33.64, & 116.86 & 43 & 212 & unknown & - & $\begin{array}{l}360 \\
\text { Up } \\
270\end{array}$ & $\begin{array}{l}.141 \\
.083 \\
.100\end{array}$ & $\begin{array}{c}0.4 \\
- \\
1 \text { peak }\end{array}$ \\
\hline 5222 & Tripp Flats & 33.60, & 116.74 & 43 & 196 & $189 / 09: 20: 53.98$ & 4.5 & $\begin{array}{l}360 \\
\text { Up } \\
270\end{array}$ & $\begin{array}{l}.051 \\
.054 \\
.076\end{array}$ & - \\
\hline 5067 & Indio & 33.747 , & 116.214 & 44 & 123 & $189 / 09: 20: 53.20$ & 6.2 & $\begin{array}{l}315 \\
\text { Up } \\
225\end{array}$ & $\begin{array}{l}.126 \\
.093 \\
.061\end{array}$ & $\begin{array}{c}0.4 \\
- \\
-\end{array}$ \\
\hline 5223 & Pine Meadows & 33.578 , & 116.589 & 44 & 177 & 189/09:20:52.91 & 5.55 & $\begin{array}{l}360 \\
U p \\
270\end{array}$ & $\begin{array}{l}.080 \\
.078 \\
.101\end{array}$ & $\overline{-}$ \\
\hline 5230 & $\begin{array}{l}\text { Santa Rosa } \\
\text { Mountain }\end{array}$ & 33.57 , & 116.52 & 45 & 169 & $189 / 09: 20: 53.25$ & 5.85 & $\begin{array}{l}360 \\
U p \\
270\end{array}$ & $\begin{array}{l}.088 \\
.057 \\
.120\end{array}$ & 1 peak \\
\hline
\end{tabular}


Table 1.--Main-shock accelerograph data for USGS stations in the greater Palm Springs region--Continued

\begin{tabular}{|c|c|c|c|c|c|c|c|c|c|c|c|}
\hline \multicolumn{4}{|c|}{ Station identification } & \multirow{2}{*}{$\begin{array}{c}\text { Epicentral } \\
\text { distance } \\
(\mathrm{km})\end{array}$} & \multirow{2}{*}{$\begin{array}{l}\text { Azimuth } \\
\text { (degrees) }\end{array}$} & \multirow{2}{*}{$\begin{array}{l}\text { Trigger } \\
\text { time } \\
\text { (G.m.t.) }\end{array}$} & \multirow{2}{*}{$\begin{array}{c}\text { S-t } \\
\text { interval } \\
\text { (s) }\end{array}$} & \multicolumn{4}{|c|}{ Acceleration } \\
\hline Number & Name & $\begin{array}{l}\text { Coor } \\
\text { (1at. }{ }^{\circ} \text { N., }\end{array}$ & $\begin{array}{l}\text { dinates } \\
\left.\text { long. }{ }^{\circ} \text { W. }\right)\end{array}$ & & & & & & $\begin{array}{l}\text { irection } \\
\text { degrees) }\end{array}$ & $\begin{array}{l}\text { Maximum } \\
(\mathrm{g})\end{array}$ & $\begin{array}{l}\text { Duration } \\
\text { (s) }\end{array}$ \\
\hline 5160 & Anza Fire Station & 33.556 , & 116.673 & 46 & 187 & $189 / 09: 20: 54.36$ & 5.1 & & $\begin{array}{l}315 \\
\text { Up } \\
225\end{array}$ & $\begin{array}{l}.068 \\
.066 \\
.114\end{array}$ & $\begin{array}{l}- \\
- \\
0.2\end{array}$ \\
\hline 5162 & $\begin{array}{l}\text { Mentone } \\
\text { Fire Station }\end{array}$ & 34.067 , & 117.117 & 48 & 283 & $189 / 09: 20: 53.02$ & 6.2 & & $\begin{array}{l}315 \\
\text { Up } \\
225\end{array}$ & $\begin{array}{l}.062 \\
.038 \\
.045\end{array}$ & - \\
\hline 5038 & Sunnymead & 33.95, & 117.15 & 50 & 268 & $189 / 09: 20: 53.54$ & 6.2 & & $\begin{array}{l}315 \\
\text { Up } \\
225\end{array}$ & $\begin{array}{l}.125 \\
.062 \\
.105\end{array}$ & $\begin{array}{c}0.2 \\
\overline{-} \\
\text { peak }\end{array}$ \\
\hline 5045 & Terwilliger Valley & 33.48 & 116.59 & 54 & 178 & unknown & 6.5 & & $\begin{array}{l}135 \\
\text { Up } \\
045\end{array}$ & $\begin{array}{l}.033 \\
.037 \\
.067\end{array}$ & - \\
\hline 5037 & $\begin{array}{l}\text { Reche Canyon } \\
\text { Olive De11 Ranch }\end{array}$ & 34.01 , & 117.22 & 56 & 275 & $189 / 09: 20: 56.78$ & 5.5 & & $\begin{array}{l}330 \\
U p \\
240\end{array}$ & $\begin{array}{l}.034 \\
.021 \\
.028\end{array}$ & $\overline{-}$ \\
\hline 5231 & Tule Canyon & 33.47, & 116.64 & 56 & 183 & unknown & 6.9 & & $\begin{array}{l}360 \\
\text { Up } \\
270\end{array}$ & $\begin{array}{l}.103 \\
.043 \\
.107\end{array}$ & $\begin{array}{l}1 \text { peak } \\
\text { peak }\end{array}$ \\
\hline \multirow[t]{2}{*}{5229} & $\begin{array}{l}\text { Loma Linda VA: } \\
\text { South freefield }\end{array}$ & 34.039 , & 117.227 & 58 & 280 & unknown & 6 & & $\begin{array}{l}360 \\
U p \\
270\end{array}$ & $\begin{array}{l}.044 \\
.027 \\
.049\end{array}$ & $\begin{array}{l}- \\
-\end{array}$ \\
\hline & North freefield & 34.059 , & 117.227 & & & $189 / 09: 20: 56.67$ & 7.1 & & $\begin{array}{l}360 \\
\text { Up } \\
270\end{array}$ & $\begin{array}{l}.052 \\
.034 \\
.044\end{array}$ & $\overline{-}$ \\
\hline 5161 & $\begin{array}{l}\text { Highland } \\
\text { Fire Station }\end{array}$ & 34.136 & 117.213 & 59 & 289 & $189 / 09: 20: 54.86$ & 7.0 & & $\begin{array}{l}315 \\
\text { Up } \\
225\end{array}$ & $\begin{array}{l}.038 \\
.045 \\
.056\end{array}$ & - \\
\hline 0129 & $\begin{array}{l}\text { Loma Linda } \\
\text { University } \\
\text { Medical Center }\end{array}$ & 34.05, & 117.26 & 61 & 279 & unknown & 6 & & $\begin{array}{l}090 \\
\text { Up } \\
360\end{array}$ & $\begin{array}{l}.036 \\
.019 \\
.049\end{array}$ & $\overline{-}$ \\
\hline 5229 & $\begin{array}{l}\text { Loma Linda VA } \\
\text { Note: } \\
\text { First floor } \\
\text { level (lst) } \\
\text { Fourth floor } \\
\text { level ( } 4 \text { th) } \\
\text { North (N) and } \\
\text { South end (S) } \\
\text { Center area (C) }\end{array}$ & 34.049 , & 117.227 & 61 & 279 & unknown & 6.3 & $\begin{array}{l}\text { 1) } \\
\text { 2) } \\
\text { 3) } \\
\text { 4) } \\
\text { 5) } \\
\text { 6) } \\
\text { 7) } \\
\text { 8) } \\
\text { 9) }\end{array}$ & $\begin{array}{l}\text { Up-1st (C) } \\
360-1 \mathrm{st}(C) \\
090-1 \mathrm{st}(C) \\
090-4 \operatorname{th}(C) \\
090-1 \mathrm{st}(\mathrm{N}) \\
360-4 \operatorname{th}(\mathrm{C}) \\
090-4 \operatorname{th}(N) \\
360-1 \mathrm{st}(\mathrm{S}) \\
090-4 \operatorname{th}(\mathrm{S})\end{array}$ & $\left\{\begin{array}{l}.017 \\
.044 \\
.044 \\
.097 \\
.044 \\
.082 \\
.092 \\
.044 \\
.108\end{array}\right.$ & $\begin{array}{l}- \\
- \\
\text { l peak } \\
- \\
- \\
- \\
\text { - } \\
1 \text { peak }\end{array}$ \\
\hline 720 & $\begin{array}{l}\text { Skinner Dam } \\
\text { Abutment }\end{array}$ & 33.58 , & 117.07 & 61 & 225 & unknown & 4.9 & & $\begin{array}{l}178 \\
\text { Up } \\
088\end{array}$ & $\begin{array}{l}.078 \\
.037 \\
.083\end{array}$ & $\begin{array}{l}- \\
-\end{array}$ \\
\hline & $\begin{array}{l}\text { Note: } \\
\text { Center crest (CC) } \\
\text { Lower crest } \\
\text { Lower slope } \\
\text { (LS) } \\
\text { Center slope } \\
\text { Center toe } \\
\text { (CT) }\end{array}$ & & & & & unknown & - & $\begin{array}{l}\text { 1) } \\
2) \\
3) \\
4) \\
5) \\
6) \\
7) \\
8) \\
9) \\
10) \\
11) \\
12)\end{array}$ & $\begin{cases}180 & (C C) \\
U p & (C C) \\
270 & (C C) \\
180 & (L C) \\
270 & (L C) \\
270 & (L S) \\
180 & (C S) \\
U p & (C S) \\
270 & (C S) \\
180 & (C T) \\
\text { Up (CT) } & \text { (CT) } \\
270 & (C T)\end{cases}$ & $\begin{array}{l}.087 \\
.053 \\
.117 \\
.071 \\
.065 \\
.055 \\
.068 \\
.059 \\
.080 \\
.097 \\
.052 \\
.086\end{array}$ & $\begin{array}{l}- \\
\text { I peak } \\
- \\
- \\
- \\
- \\
- \\
- \\
\text { peak } \\
- \\
-\end{array}$ \\
\hline
\end{tabular}


Table 1.--Main-shock accelerograph data for USGS stations in the greater Palm Springs region--Continued

\begin{tabular}{|c|c|c|c|c|c|c|c|c|c|c|}
\hline \multicolumn{4}{|c|}{ Station identification } & \multirow{2}{*}{$\begin{array}{c}\text { Epicentral } \\
\text { distance } \\
(\mathrm{km})\end{array}$} & \multirow{2}{*}{$\begin{array}{c}\text { Azimuth } \\
\text { (degrees) }\end{array}$} & \multirow{2}{*}{$\begin{array}{l}\text { Trigger } \\
\text { time } \\
\text { (G.m.t.) }\end{array}$} & \multirow{2}{*}{$\begin{array}{c}\text { S-t } \\
\text { interval } \\
(s)\end{array}$} & \multicolumn{3}{|c|}{ Acceleration } \\
\hline Number & Name & $\begin{array}{l}\text { Coor } \\
\text { (lat. }{ }^{\circ} \text {., }\end{array}$ & $\begin{array}{l}\text { dinates } \\
\left.\text { long. }{ }^{\circ} \text { W. }\right)\end{array}$ & & & & & $\begin{array}{l}\text { Direction } \\
\text { (degrees) }\end{array}$ & $\begin{array}{l}\text { Maximum } \\
(\mathrm{g})\end{array}$ & $\begin{array}{l}\text { Duration } \\
\text { (s) }\end{array}$ \\
\hline \multirow[t]{2}{*}{754} & $\begin{array}{l}\text { Colton Interchange: } \\
\text { Bridge cell }\end{array}$ & : 34.06 & 117.30 & 64 & 279 & unknown & 6.4 & $\begin{array}{l}082 \\
\text { Up } \\
352\end{array}$ & $\begin{array}{l}.122 \\
.046 \\
.100\end{array}$ & $\begin{array}{c}1.7 \\
1 \text { peak }\end{array}$ \\
\hline & Vault & & & & & unknown & 5.9 & $\begin{array}{l}082 \\
\text { Up } \\
352\end{array}$ & $\begin{array}{l}.058 \\
.017 \\
.056\end{array}$ & - \\
\hline 5221 & Chinuahua valley & 33.38, & 116.68 & 66 & 186 & unknown & 7.9 & $\begin{array}{l}270 \\
U p \\
18 \sigma\end{array}$ & $\begin{array}{l}.050 \\
.038 \\
.067\end{array}$ & - \\
\hline 5047 & Rancho de Anza & 33.35, & 116.40 & 72 & 164 & unknown & 8.2 & $\begin{array}{l}135 \\
\text { Up } \\
045\end{array}$ & $\begin{array}{l}.043 \\
.027 \\
.050\end{array}$ & $\begin{array}{l}- \\
-\end{array}$ \\
\hline 0707 & $\begin{array}{l}\text { Lake Mathews } \\
\text { Dike toe }\end{array}$ & 33.852 , & 117.451 & 79 & 261 & unknown & 6.8 & $\begin{array}{l}252 \\
\text { Up } \\
162\end{array}$ & $\begin{array}{l}.056 \\
.041 \\
.066\end{array}$ & - \\
\hline 5220 & Borrego Springs & 33.21, & 116.33 & 88 & 163 & $189 / 09: 21: 02.5$ & 9.3 & $\begin{array}{l}315 \\
\text { Up } \\
225\end{array}$ & $\begin{array}{l}.016 \\
.016 \\
.017\end{array}$ & $\begin{array}{l}- \\
-\end{array}$ \\
\hline 5035 & Lytle Creek & 34.26 , & 117.50 & 88 & 292 & $189 / 09: 21: 11.98$ & - & $\begin{array}{l}315 \\
\text { Up } \\
225\end{array}$ & $\begin{array}{l}.021 \\
.016 \\
.022\end{array}$ & $\begin{array}{l}- \\
-\end{array}$ \\
\hline \multirow[t]{3}{*}{969} & $\begin{array}{l}\text { Prado Dam: } \\
\text { Abutment }\end{array}$ & 33.89, & 117.64 & 95 & 265 & unknown & - & $\begin{array}{l}090 \\
U p \\
360\end{array}$ & $\begin{array}{l}.017 \\
.010 \\
.023\end{array}$ & - \\
\hline & Downstream & & & & & unknown & 11.0 & $\begin{array}{l}090 \\
\text { Up } \\
360\end{array}$ & $\begin{array}{l}.053 \\
.039 \\
.053\end{array}$ & $\begin{array}{l}- \\
-\end{array}$ \\
\hline & Crest & & & & & unknown & - & $\begin{array}{l}090 \\
\text { Up } \\
360\end{array}$ & $\begin{array}{l}.025 \\
.017 \\
.028\end{array}$ & - \\
\hline 5034 & Lone Pine Canyon & 34.32 , & 117.57 & 96 & 294 & $189 / 09: 21: 03.65$ & 8.7 & $\begin{array}{l}120 \\
\text { Up } \\
030\end{array}$ & $\begin{array}{l}.027 \\
.010 \\
.016\end{array}$ & - \\
\hline \multirow[t]{2}{*}{287} & $\begin{array}{l}\text { San Antonio Dam: } \\
\text { Right abutment }\end{array}$ & 34.16, & 117.68 & 101 & 282 & unknown & - & $\begin{array}{l}090 \\
\text { Up } \\
360\end{array}$ & $\begin{array}{l}.010 \\
.011 \\
.005\end{array}$ & - \\
\hline & Crest & & & & & unknown & - & $\begin{array}{l}090 \\
\text { Up } \\
360\end{array}$ & $\begin{array}{l}.017 \\
.011 \\
.027\end{array}$ & $\overline{-}$ \\
\hline 5033 & Big Pines Station & 34.38, & 117.69 & 109 & 295 & $189 / 09: 21: 16.73$ & - & $\begin{array}{l}300 \\
\text { Up } \\
210\end{array}$ & $\begin{array}{l}.021 \\
.010 \\
.026\end{array}$ & - \\
\hline \multirow[t]{2}{*}{698} & $\begin{array}{l}\text { Diemer Filter } \\
\text { Plant: } \\
\text { Basement }\end{array}$ & 33.91, & 117.82 & 112 & 267 & unknown & 11.2 & $\begin{array}{l}281 \\
\text { Up } \\
191\end{array}$ & $\begin{array}{l}.017 \\
.010 \\
.022\end{array}$ & $\begin{array}{l}- \\
-\end{array}$ \\
\hline & Reservoir roof & & & & & uniknown & 11.6 & $\begin{array}{l}281 \\
\text { Up } \\
191\end{array}$ & $\begin{array}{l}.021 \\
.022 \\
.034\end{array}$ & $\begin{array}{l}- \\
-\end{array}$ \\
\hline
\end{tabular}


Table 1.--Main-shock accelerograph data for USGS stations in the greater Palm Springs region--Continued

\begin{tabular}{|c|c|c|c|c|c|c|c|c|c|c|}
\hline \multicolumn{4}{|c|}{ Station identification } & \multirow{2}{*}{$\begin{array}{c}\text { Epicentral } \\
\text { distance } \\
(\mathrm{km})\end{array}$} & \multirow{2}{*}{$\begin{array}{l}\text { Azimuth } \\
\text { (degrees) }\end{array}$} & \multirow{2}{*}{$\begin{array}{l}\text { Trigger } \\
\text { time } \\
\text { (G.m.t.) }\end{array}$} & \multirow{2}{*}{$\begin{array}{c}S-t \\
\text { interval } \\
(s)\end{array}$} & \multicolumn{3}{|c|}{ Acceleration } \\
\hline Number & Name & $\begin{array}{l}\text { Coorc } \\
\text { (lat. }{ }^{\circ} \text { N., }\end{array}$ & $\begin{array}{l}\text { dinates } \\
\left.\text { long. }{ }^{\circ} \mathrm{W.}_{\text {. }}\right)\end{array}$ & & & & & $\begin{array}{l}\text { Direction } \\
\text { (degrees) }\end{array}$ & $\begin{array}{l}\text { Maximum } \\
(\mathrm{g})\end{array}$ & $\begin{array}{l}\text { Duration } \\
\text { (s) }\end{array}$ \\
\hline 108 & $\begin{array}{l}\text { Carbon Canyon Dam } \\
\text { Crest }\end{array}$ & 33.92 , & 117.84 & 114 & 268 & unknown & - & $\begin{array}{l}130 \\
\text { Up } \\
040\end{array}$ & $\begin{array}{l}.026 \\
.015 \\
.026\end{array}$ & - \\
\hline \multirow[t]{3}{*}{951} & $\begin{array}{l}\text { Brea Dam: } \\
\text { Left abutment }\end{array}$ & 33.890 & 117.930 & 122 & 266 & unknown & 14.3 & $\begin{array}{l}130 \\
\text { Up } \\
040\end{array}$ & $\begin{array}{l}.026 \\
.024 \\
.037\end{array}$ & $\begin{array}{l}- \\
-\end{array}$ \\
\hline & Downstream & & & & & unknown & 14.1 & $\begin{array}{l}130 \\
\text { Up } \\
040\end{array}$ & $\begin{array}{l}.046 \\
.020 \\
.040\end{array}$ & $\begin{array}{l}- \\
-\end{array}$ \\
\hline & Crest & & & & & unknown & 14.0 & $\begin{array}{l}130 \\
\text { Up } \\
040\end{array}$ & $\begin{array}{l}.041 \\
.032 \\
.070\end{array}$ & $\begin{array}{l}- \\
-\end{array}$ \\
\hline \multirow[t]{2}{*}{5164} & $\begin{array}{l}\text { Weymouth } \\
\text { Filter Plant: } \\
\text { Ground }\end{array}$ & 34.506 , & 117.778 & 123 & 299 & unknown & 9.7 & $\begin{array}{l}017 \\
\text { Up } \\
287\end{array}$ & $\begin{array}{l}.020 \\
.011 \\
.021\end{array}$ & $\begin{array}{l}- \\
-\end{array}$ \\
\hline & Tank & & & & & unknown & 9.7 & $\begin{array}{l}017 \\
\text { Up } \\
287\end{array}$ & $\begin{array}{l}.064 \\
.045 \\
.077\end{array}$ & $\overline{-}$ \\
\hline \multirow[t]{3}{*}{804} & $\begin{array}{l}\text { Whittier } \\
7215 \text { Bright Ave: } \\
\text { Basement }\end{array}$ & 33.977 , & 118.036 & 131 & 271 & unknown & - & $\begin{array}{l}180 \\
\text { Up } \\
090\end{array}$ & $\begin{array}{l}.017 \\
.011 \\
.026\end{array}$ & $\begin{array}{l}- \\
-\end{array}$ \\
\hline & 5th fioor & & & & & unknown & - & $\begin{array}{l}180 \\
\text { Up } \\
090\end{array}$ & $\begin{array}{l}.042 \\
.016 \\
.056\end{array}$ & $\begin{array}{l}- \\
-\end{array}$ \\
\hline & loth floor & & & & & unknown & - & $\begin{array}{l}180 \\
\text { Up } \\
090\end{array}$ & $\begin{array}{l}.042 \\
.022 \\
.079\end{array}$ & $\begin{array}{l}- \\
-\end{array}$ \\
\hline \multirow[t]{5}{*}{634} & $\begin{array}{l}\text { Norwalk, } 12400 \\
\text { Imperial Highway: } \\
\text { Basement }\end{array}$ & 33.92 , & 118.07 & 135 & 268 & unknown & 16.8 & $\begin{array}{l}090 \\
\text { Up } \\
360\end{array}$ & $\begin{array}{l}.030 \\
.015 \\
.034\end{array}$ & $\begin{array}{l}- \\
-\end{array}$ \\
\hline & 4 th floor & & & & & unknown & 16.8 & $\begin{array}{l}090 \\
\text { Up } \\
360\end{array}$ & $\begin{array}{l}.041 \\
.020 \\
.048\end{array}$ & $\begin{array}{l}- \\
-\end{array}$ \\
\hline & 8th floor(roof) & & & & & unknown & 16.9 & $\begin{array}{l}090 \\
\text { Up } \\
360\end{array}$ & $\begin{array}{l}.063 \\
.024 \\
.079\end{array}$ & $\begin{array}{l}- \\
-\end{array}$ \\
\hline & North freefield & & & & & unknown & 16.8 & $\begin{array}{l}090 \\
\text { Up } \\
360\end{array}$ & $\begin{array}{l}.041 \\
.024 \\
.048\end{array}$ & $\begin{array}{l}- \\
-\end{array}$ \\
\hline & South freefield & & & & & unknown & 16.6 & $\begin{array}{l}090 \\
\text { Up } \\
360\end{array}$ & $\begin{array}{l}.033 \\
.017 \\
.043\end{array}$ & $\begin{array}{l}- \\
-\end{array}$ \\
\hline \multirow[t]{3}{*}{5239} & $\begin{array}{l}\text { Norwalk, } 12440 \\
\text { Imperial Highway: } \\
\text { Basement }\end{array}$ & 33.92 & 118.07 & 135 & 268 & unknown & 16.8 & $\begin{array}{l}090 \\
\text { Up } \\
360\end{array}$ & $\begin{array}{l}.028 \\
.027 \\
.041\end{array}$ & $\overline{-}$ \\
\hline & North freefield & & & & & unknown & 16.8 & $\begin{array}{l}090 \\
\text { Up } \\
360\end{array}$ & $\begin{array}{l}.033 \\
.016 \\
.033\end{array}$ & $\begin{array}{l}- \\
-\end{array}$ \\
\hline & South freefield & & & & & unknown & 16.8 & $\begin{array}{l}090 \\
\text { Up } \\
360\end{array}$ & $\begin{array}{l}.027 \\
.016 \\
.041\end{array}$ & $\begin{array}{l}- \\
-\end{array}$ \\
\hline
\end{tabular}


Table 1.--Main-shock accelerograph data for USGS stations in the greater Palm Springs region--Continued

\begin{tabular}{|c|c|c|c|c|c|c|c|c|c|c|}
\hline \multicolumn{4}{|c|}{ Station identification } & \multirow{2}{*}{$\begin{array}{l}\text { Epicentral } \\
\text { distance } \\
(\mathrm{km})\end{array}$} & \multirow{2}{*}{$\begin{array}{c}\text { Azimuth } \\
\text { (degrees) }\end{array}$} & \multirow{2}{*}{$\begin{array}{l}\text { Trigger } \\
\text { time } \\
\text { (G.m.t.) }\end{array}$} & \multirow{2}{*}{$\begin{array}{l}\text { s-t } \\
\text { interval } \\
(s)\end{array}$} & \multicolumn{3}{|c|}{ Acceleration } \\
\hline Number & Name & $\begin{array}{l}\text { Coor } \\
\text { (1at. }{ }^{\circ} \mathrm{N}, \text {, }\end{array}$ & $\begin{array}{l}\text { dinates } \\
\left.\text { long. }{ }^{\circ} W_{.}\right)\end{array}$ & & & & & $\begin{array}{l}\text { Direction } \\
\text { (degrees) }\end{array}$ & $\begin{array}{l}\text { Maximum } \\
(\mathrm{g})\end{array}$ & $\begin{array}{l}\text { Duration } \\
\text { (s) }\end{array}$ \\
\hline 5239 & $\begin{array}{l}\text { Norwalk, } 12440 \\
\text { Imperial Highway } \\
\text { Bechtel Bldg. } 43 \\
\text { Note: } \\
\text { Basement level (B } \\
\text { First floor level } \\
\text { Second floor leve } \\
\text { Fifth floor level } \\
\text { Seventh floor lev } \\
30 \mathrm{ft} \text {. downhole }\end{array}$ & $\begin{array}{l}33.92, \\
\\
\text { Bsmt) } \\
1 \text { (1st) } \\
\text { el (2nd) } \\
\text { (5th) } \\
\text { vel (7th) } \\
\text { level (DH) }\end{array}$ & 118.07 & 135 & 268 & $189 / 09: 21: 08.15$ & 16.8 & $\begin{array}{l}\text { 1) } 090 \text { (7th) } \\
\text { 2) } 090 \text { (5th) } \\
\text { 3) } 090 \text { (2nd) } \\
\text { 4) } 090 \text { (1st) } \\
\text { 5) } 360 \text { (Bsmt) } \\
\text { 6) } 360 \text { (5th) } \\
\text { 7) } 090 \text { (Bsmt) } \\
\text { 8) Up (Bsmt) } \\
\text { 9) } 360(\mathrm{Bsmt}) \\
\text { 10) } 090(\mathrm{DH}) \\
\text { 11) Up -(DH) } \\
\text { 12) } 360(\mathrm{DH})\end{array}$ & $\left\{\begin{array}{l}.057 \\
.049 \\
.050 \\
.041 \\
.031 \\
.045 \\
.010 \\
.027 \\
.041 \\
.011 \\
.021 \\
.026\end{array}\right.$ & $\begin{array}{l}- \\
- \\
- \\
- \\
- \\
- \\
- \\
-\end{array}$ \\
\hline 5129 & $\begin{array}{l}\text { Los Angeles } \\
\text { Bulk Mail } \\
\text { Facility }\end{array}$ & 33.99 , & 118.16 & 143 & 271 & unknown & 16.5 & $\begin{array}{l}010 \\
\text { Up } \\
280\end{array}$ & $\begin{array}{l}.033 \\
.011 \\
.027\end{array}$ & - \\
\hline \multirow[t]{3}{*}{655} & $\begin{array}{l}\text { Jensen Filter } \\
\text { Plant: } \\
\text { Admin. Building } \\
\text { basement }\end{array}$ & 34.31, & 118.50 & 175 & 275 & unknown & 18.5 & $\begin{array}{l}025 \\
\text { Up } \\
295\end{array}$ & $\begin{array}{l}.011 \\
.006 \\
.012\end{array}$ & $\begin{array}{l}- \\
-\end{array}$ \\
\hline & $\begin{array}{l}\text { Generator room } \\
\text { basement }\end{array}$ & & & & & unknown & 18.8 & $\begin{array}{l}025 \\
\text { Up } \\
295\end{array}$ & $\begin{array}{l}.016 \\
.005 \\
.011\end{array}$ & $\begin{array}{l}- \\
-\end{array}$ \\
\hline & Reservoir roof & & & & & unknown & 18.0 & $\begin{array}{l}025 \\
\text { Up } \\
295\end{array}$ & $\begin{array}{l}.011 \\
.006 \\
.017\end{array}$ & $\begin{array}{l}- \\
-\end{array}$ \\
\hline 710 & $\begin{array}{l}\text { Palos Verdes } \\
\text { Reservoir }\end{array}$ & 33.77 , & 118.32 & 180 & 300 & unknown & - & $\begin{array}{l}165 \\
\text { Up } \\
075\end{array}$ & $\begin{array}{l}.021 \\
.016 \\
.021\end{array}$ & $\begin{array}{l}- \\
-\end{array}$ \\
\hline
\end{tabular}


Table 2.- Aftershock accelerograph data for USGS stations in the greater Palm Springs region

[Trigger time includes day (beginning with July 8, day number 189)/hour:minute:second; time in parentheses indicates P-wave arrival time. S-t interval is time between instrument triggering and arrival of S-wave motion; interval in parentheses indicates time between arrivals of $P_{-}$and S-wave motion; dash indicates interval is indeterminable. Direction of acceleratior is for upward trace deflection on accelerogram; vertical-component directions are listed as "up" or "down". Duration of acceleration is time between first and last peaks of acceleration greater than $0.10 \mathrm{~g}$.

\begin{tabular}{|c|c|c|c|c|c|c|c|c|c|}
\hline \multirow{2}{*}{$\begin{array}{l}\text { Trigger time } \\
\text { (G.m.t.) }\end{array}$} & \multirow{2}{*}{$\begin{array}{c}S-t \\
\text { interval } \\
(s)\end{array}$} & \multicolumn{3}{|c|}{ Acceleration } & \multirow{2}{*}{$\begin{array}{l}\text { Trigger time } \\
\text { (G.m.t.) }\end{array}$} & \multirow{2}{*}{$\begin{array}{c}\text { S-t } \\
\text { interval } \\
(s)\end{array}$} & \multicolumn{3}{|c|}{ Acceleration } \\
\hline & & $\begin{array}{l}\text { Direction } \\
\text { (degrees) }\end{array}$ & $\begin{array}{l}\text { Maximum } \\
(\mathrm{g})\end{array}$ & $\begin{array}{l}\text { Duration } \\
\text { (s) }\end{array}$ & & & $\begin{array}{l}\text { Direction } \\
\text { (degrees) }\end{array}$ & $\begin{array}{l}\text { Maximum } \\
(\mathrm{g})\end{array}$ & $\begin{array}{l}\text { Duration } \\
\text { (s) }\end{array}$ \\
\hline & Whitewater & $r$ Trout Far & & & Unknown & 1.65 & $\begin{array}{l}270 \\
\text { Up } \\
180\end{array}$ & $\begin{array}{l}.022 \\
.022 \\
.033\end{array}$ & $\begin{array}{l}--- \\
--- \\
---\end{array}$ \\
\hline $\begin{array}{l}\text { (Unknown, } \\
52.35 \text { s. after } \\
\text { main-shock trigger.) }\end{array}$ & $(1.8)$ & $\begin{array}{l}270 \\
\text { Up } \\
180\end{array}$ & $\begin{array}{l}.043 \\
.027 \\
.028\end{array}$ & $\begin{array}{l}--- \\
--- \\
--\end{array}$ & Unknown & - & $\begin{array}{l}270 \\
\text { Up } \\
180\end{array}$ & $\begin{array}{l}.027 \\
.016 \\
.028\end{array}$ & $\begin{array}{l}--- \\
--- \\
--\end{array}$ \\
\hline Unknown & 1.8 & $\begin{array}{l}270 \\
\text { Up } \\
180\end{array}$ & $\begin{array}{l}.054 \\
.054 \\
.050\end{array}$ & $\begin{array}{l}--- \\
--- \\
--\end{array}$ & Unknown & 1.5 & $\begin{array}{l}270 \\
\text { Up } \\
180\end{array}$ & $\begin{array}{l}.043 \\
.038 \\
.044\end{array}$ & $\begin{array}{l}--- \\
--- \\
--\end{array}$ \\
\hline (Unknown) & $(0.2)$ & $\begin{array}{l}270 \\
\text { Up } \\
180\end{array}$ & $\begin{array}{l}.005 \\
.011 \\
.006\end{array}$ & $\begin{array}{l}-- \\
-- \\
--\end{array}$ & Unknown & - & $\begin{array}{l}270 \\
\text { Up } \\
180\end{array}$ & $\begin{array}{l}.032 \\
.027 \\
.066\end{array}$ & $\begin{array}{l}--- \\
--- \\
---\end{array}$ \\
\hline Unknown & 1.6 & $\begin{array}{l}270 \\
\text { Up } \\
180\end{array}$ & $\begin{array}{l}.032 \\
.038 \\
.072\end{array}$ & $\begin{array}{l}--- \\
--- \\
--\end{array}$ & Unknown & 0.55 & $\begin{array}{l}270 \\
\text { Up } \\
180\end{array}$ & $\begin{array}{l}.027 \\
.032 \\
.072\end{array}$ & $\begin{array}{l}--- \\
--- \\
--\end{array}$ \\
\hline (Unknown) & $(1.8)$ & $\begin{array}{l}270 \\
\text { Up } \\
180\end{array}$ & $\begin{array}{l}.005 \\
.005 \\
.006\end{array}$ & $\begin{array}{l}--- \\
--- \\
--\end{array}$ & Unknown & 0.65 & $\begin{array}{l}270 \\
\text { Up } \\
180\end{array}$ & $\begin{array}{l}.081 \\
.032 \\
.083\end{array}$ & $\begin{array}{l}--- \\
--- \\
--\end{array}$ \\
\hline Unknown & 1.35 & $\begin{array}{l}270 \\
\text { Up } \\
180\end{array}$ & $\begin{array}{l}.016 \\
.022 \\
.039\end{array}$ & $\begin{array}{l}--- \\
--- \\
--\end{array}$ & $192 / 08: 51: 33.05$ & - & $\begin{array}{l}270 \\
\text { Up } \\
180\end{array}$ & $\begin{array}{l}.011 \\
.011 \\
.011\end{array}$ & $\begin{array}{l}--- \\
--- \\
---\end{array}$ \\
\hline (Unknown) & $(1.7)$ & $\begin{array}{l}270 \\
\text { Up } \\
180\end{array}$ & $\begin{array}{l}.016 \\
.016 \\
.0111\end{array}$ & $\begin{array}{l}--- \\
--- \\
---\end{array}$ & Unknown & 0.9 & $\begin{array}{l}270 \\
\text { Up } \\
180\end{array}$ & $\begin{array}{l}.043 \\
.027 \\
.033\end{array}$ & $\begin{array}{l}--- \\
--- \\
--\end{array}$ \\
\hline Unknown & 0.35 & $\begin{array}{l}270 \\
\text { Up } \\
180\end{array}$ & $\begin{array}{l}.032 \\
.038 \\
.044\end{array}$ & $\begin{array}{l}--- \\
--- \\
--\end{array}$ & Unknown & 1.7 & $\begin{array}{l}270 \\
\text { Up } \\
180\end{array}$ & $\begin{array}{l}.022 \\
.022 \\
.017\end{array}$ & $\begin{array}{l}--- \\
--- \\
---\end{array}$ \\
\hline Unknown & - & $\begin{array}{l}270 \\
\text { Up } \\
180\end{array}$ & $\begin{array}{l}.032 \\
.016 \\
.028\end{array}$ & $\begin{array}{l}--- \\
--- \\
---\end{array}$ & Unknown & 0.3 & $\begin{array}{l}270 \\
\text { Up } \\
180\end{array}$ & $\begin{array}{l}.011 \\
.011 \\
.017\end{array}$ & $\begin{array}{l}--- \\
--- \\
--\end{array}$ \\
\hline Unknown & - & $\begin{array}{l}270 \\
\text { Up } \\
180\end{array}$ & $\begin{array}{l}.016 \\
.016 \\
.017\end{array}$ & $\begin{array}{l}--- \\
--- \\
---\end{array}$ & Unknown & 0.4 & $\begin{array}{l}270 \\
\text { Up } \\
180\end{array}$ & $\begin{array}{l}.011 \\
.011 \\
.017\end{array}$ & $\begin{array}{l}--- \\
--- \\
---\end{array}$ \\
\hline Unknown & - & $\begin{array}{l}270 \\
\text { Up } \\
180\end{array}$ & $\begin{array}{l}.038 \\
.027 \\
.028\end{array}$ & $\begin{array}{l}--- \\
--- \\
---\end{array}$ & $198 / 20: 35: 16.33$ & 1.2 & $\begin{array}{l}270 \\
\text { Up } \\
180\end{array}$ & $\begin{array}{l}.135 \\
.081 \\
.105\end{array}$ & $\begin{array}{l}\text { I peak } \\
-\overline{0.05}\end{array}$ \\
\hline Unknown & 1.3 & $\begin{array}{l}270 \\
\text { Up } \\
180\end{array}$ & $\begin{array}{l}.081 \\
.065 \\
.099\end{array}$ & $\begin{array}{l}--- \\
\bar{I} \text { peak }\end{array}$ & $198 / 21: 54: 46.55$ & 1.1 & $\begin{array}{l}270 \\
\text { Up } \\
180\end{array}$ & $\begin{array}{l}.162 \\
.081 \\
.160\end{array}$ & $\begin{array}{l}0.55 \\
--- \\
0.35\end{array}$ \\
\hline Unknown & 1.55 & $\begin{array}{l}270 \\
\text { Up } \\
180\end{array}$ & $\begin{array}{l}.038 \\
.027 \\
.039\end{array}$ & $\begin{array}{l}--- \\
--- \\
---\end{array}$ & $210 / 06: 43: 53.7$ & - & $\begin{array}{l}270 \\
\text { Up } \\
180\end{array}$ & $\begin{array}{l}.016 \\
.016 \\
.028\end{array}$ & $\begin{array}{l}--- \\
--- \\
---\end{array}$ \\
\hline Unknown & 0.8 & $\begin{array}{l}270 \\
\text { Up } \\
180\end{array}$ & $\begin{array}{l}.038 \\
.032 \\
.028\end{array}$ & $\begin{array}{l}--- \\
--- \\
---\end{array}$ & $212 / 07: 51: 47.15$ & - & $\begin{array}{l}270 \\
\text { Up } \\
180\end{array}$ & $\begin{array}{l}.011 \\
.011 \\
.011\end{array}$ & $\begin{array}{l}--- \\
--- \\
--\end{array}$ \\
\hline
\end{tabular}


Table 2.--Aftershock accelerograph data for USGS stations in the greater Palm Springs region--Continued

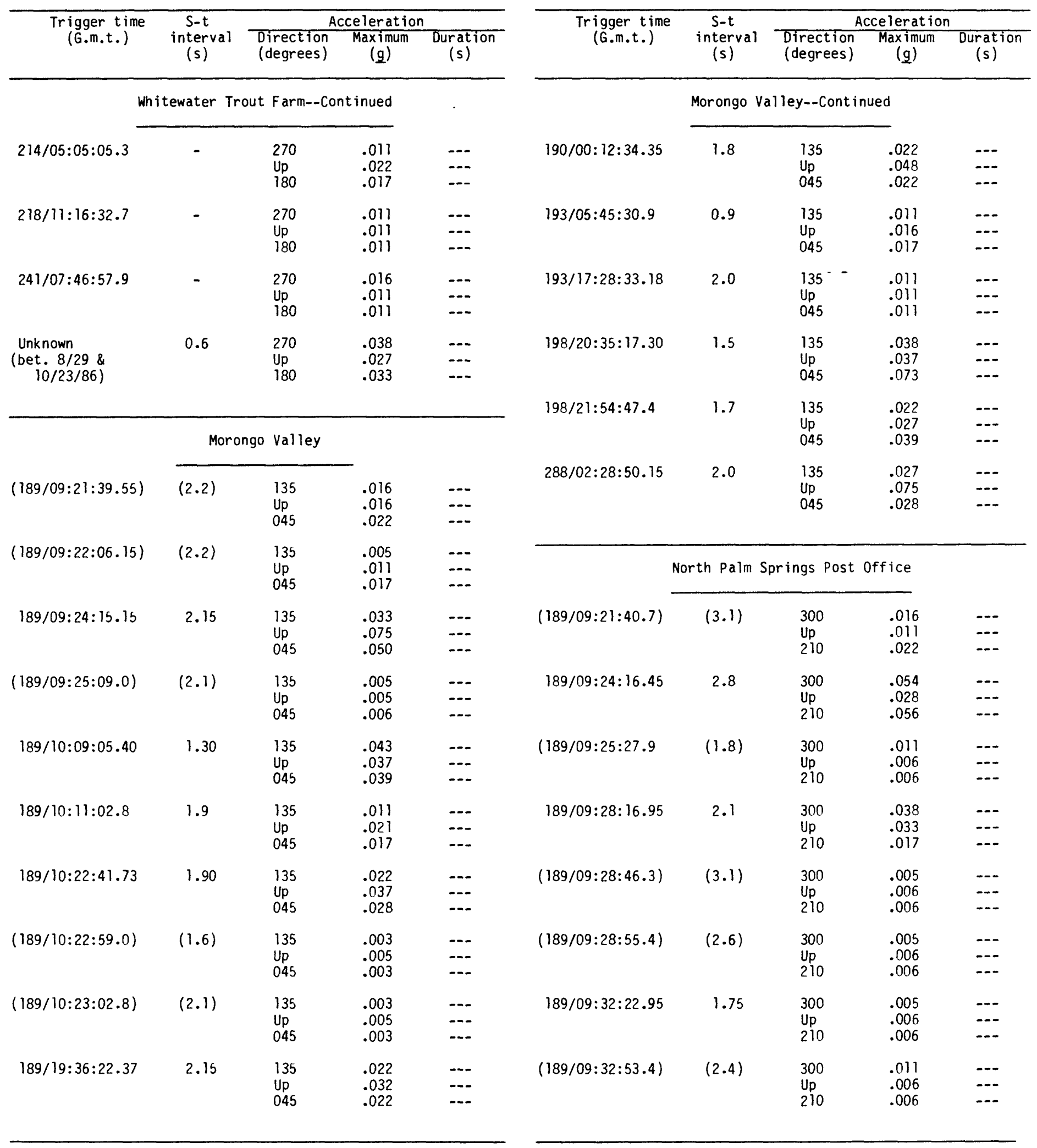


Table 2.--Aftershock accelerograph data for USGS stations in the greater Palm Springs region--Cont inued

\begin{tabular}{|c|c|c|c|c|c|c|c|c|c|}
\hline \multirow{2}{*}{$\begin{array}{c}\text { Trigger time } \\
\text { (G.m.t.) }\end{array}$} & \multirow{2}{*}{$\begin{array}{c}S-t \\
\text { interval } \\
(s)\end{array}$} & \multicolumn{3}{|c|}{ Acceleration } & \multirow{2}{*}{$\begin{array}{l}\text { Trigger time } \\
\text { (G.m.t.) }\end{array}$} & \multirow{2}{*}{$\begin{array}{c}\text { S-t } \\
\text { interval } \\
(s)\end{array}$} & \multicolumn{3}{|c|}{ Acceleration } \\
\hline & & $\begin{array}{l}\text { Direction } \\
\text { (degrees) }\end{array}$ & $\begin{array}{l}\text { Maximum } \\
(\mathrm{g})\end{array}$ & $\begin{array}{l}\text { Duration } \\
(\mathrm{s})\end{array}$ & & & $\begin{array}{l}\text { Direction } \\
\text { (degrees) }\end{array}$ & $\begin{array}{l}\text { Maximum } \\
(\mathrm{g})\end{array}$ & $\begin{array}{l}\text { Duration } \\
\text { (s) }\end{array}$ \\
\hline \multicolumn{5}{|c|}{ North Palm Springs Post Office--Continued } & \multicolumn{5}{|c|}{ North Palm Springs Post Office--Continued } \\
\hline $189 / 09: 49: 52.35$ & 2.1 & $\begin{array}{l}300 \\
\text { Up } \\
210\end{array}$ & $\begin{array}{l}.027 \\
.022 \\
.028\end{array}$ & 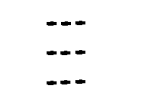 & $241 / 07: 46: 56.45$ & 1.7 & $\begin{array}{l}300 \\
\text { Up } \\
210\end{array}$ & $\begin{array}{l}.135 \\
.044 \\
.112\end{array}$ & $\begin{array}{l}0.1 \\
-1 \text { peak }\end{array}$ \\
\hline$(189 / 09: 50: 57.0)$ & $(2.5)$ & $\begin{array}{l}300 \\
\text { Up } \\
210\end{array}$ & $\begin{array}{l}.005 \\
.006 \\
.006\end{array}$ & 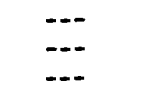 & $252 / 16: 22: 52.7$ & 1.5 & $\begin{array}{l}300 \\
\text { Up } \\
210\end{array}$ & $\begin{array}{l}.054 \\
.022 \\
.050\end{array}$ & $\begin{array}{l}--- \\
--- \\
--\end{array}$ \\
\hline $189 / 10: 04: 55.00$ & 1.4 & $\begin{array}{l}300 \\
\text { Up } \\
210\end{array}$ & $\begin{array}{l}.016 \\
.011 \\
.011\end{array}$ & $\ldots$ & $288 / 02: 28: 49.75$ & 1.5 & $\begin{array}{l}300 \\
\text { Up } \\
210\end{array}$ & $\begin{array}{l}.151 \\
.089 \\
.073\end{array}$ & $\begin{array}{l}0.3 \\
\cdots-- \\
---\end{array}$ \\
\hline $189 / 10: 09: 05.13$ & 1.7 & $\begin{array}{l}300 \\
\text { Up } \\
210\end{array}$ & $\begin{array}{l}.168 \\
.061 \\
.140\end{array}$ & $\begin{array}{l}0.1 \\
0.3\end{array}$ & & \multicolumn{2}{|c|}{ Cabazon } & & \\
\hline $189 / 10: 11: 06.33$ & 0.3 & $\begin{array}{l}300 \\
\text { Up } \\
210\end{array}$ & $\begin{array}{l}.027 \\
.017 \\
.056\end{array}$ & $\cdots$ & $(189 / 09: 21: 40)$ & $(-)$ & $\begin{array}{l}270 \\
\text { Up } \\
180\end{array}$ & $\begin{array}{l}.006 \\
.005 \\
.005\end{array}$ & $\begin{array}{l}--- \\
---\end{array}$ \\
\hline $189 / 10: 22: 42.95$ & 2.4 & $\begin{array}{l}300 \\
\text { Up } \\
210\end{array}$ & $\begin{array}{l}.027 \\
.022 \\
.039\end{array}$ & $\cdots$ & $189 / 09: 30: 27.66$ & 0.2 & $\begin{array}{l}270 \\
\text { Up } \\
180\end{array}$ & $\begin{array}{l}.023 \\
.022 \\
.027\end{array}$ &.- \\
\hline $189 / 13: 11: 44.9$ & - & $\begin{array}{l}300 \\
\text { Up } \\
210\end{array}$ & $\begin{array}{l}.005 \\
.006 \\
.006\end{array}$ & $\cdots$ & $(189 / 09: 30: 47.6)$ & $(0.4)$ & $\begin{array}{l}270 \\
\text { Up } \\
180\end{array}$ & $\begin{array}{l}.006 \\
.011 \\
.011\end{array}$ & $\begin{array}{l}--- \\
---\end{array}$ \\
\hline $189 / 16: 39: 46.99$ & 2.20 & $\begin{array}{l}300 \\
U p \\
210\end{array}$ & $\begin{array}{l}.065 \\
.011 \\
.028\end{array}$ & $\cdots$ & $189 / 10: 09: 09.28$ & 0.25 & $\begin{array}{l}270 \\
\text { Up } \\
180\end{array}$ & $\begin{array}{l}.011 \\
.016 \\
.011\end{array}$ & - \\
\hline $189 / 19: 36: 23.32$ & 2.55 & $\begin{array}{l}300 \\
\text { Up } \\
210\end{array}$ & $\begin{array}{l}.022 \\
.017 \\
.028\end{array}$ & $\cdots$ & $198 / 21: 54: 50.3$ & 0.2 & $\begin{array}{l}270 \\
\text { Up } \\
180\end{array}$ & $\begin{array}{l}.017 \\
.016 \\
.027\end{array}$ & -- \\
\hline $190 / 00: 12: 34.44$ & 1.80 & $\begin{array}{l}300 \\
\text { Up }\end{array}$ & $\begin{array}{l}.108 \\
.056\end{array}$ & 1 peak & & & & & \\
\hline & & & & peak & \multicolumn{5}{|c|}{ Fun Valley } \\
\hline \multirow[t]{2}{*}{$190 / 09: 41: 23.21$} & 1.80 & 300 & .027 & $\cdots$ & \multirow[b]{2}{*}{$(189 / 09: 21: 42)$} & & & & \\
\hline & & 210 & .022 & $\cdots$ & & $(-)$ & $\begin{array}{l}135 \\
\text { Up }\end{array}$ & $\begin{array}{l}.003 \\
.003\end{array}$ & -- \\
\hline \multirow[t]{2}{*}{$194 / 01: 41: 40.98$} & 2.1 & $\begin{array}{l}300 \\
\text { Up }\end{array}$ & $\begin{array}{l}.032 \\
.017\end{array}$ & --- & \multirow{2}{*}{ 189/09:30:29.4 } & & 045 & .003 & --- \\
\hline & & 210 & .028 & -- & & - & $\begin{array}{l}135 \\
\text { Up }\end{array}$ & $\begin{array}{l}.039 \\
.023\end{array}$ & --- \\
\hline \multirow[t]{2}{*}{$198 / 20: 35: 17.81$} & 2.2 & $\begin{array}{l}300 \\
\text { Up }\end{array}$ & $\begin{array}{l}.043 \\
.033\end{array}$ & $\cdots$ & \multirow{3}{*}{ Unknown } & & 045 & .050 & -- \\
\hline & & 210 & .078 & -- & & 2.6 & $\begin{array}{l}135 \\
\text { Up }\end{array}$ & $\begin{array}{l}.028 \\
.017\end{array}$ & --- \\
\hline $198 / 21: 54: 48.08$ & 2.2 & $\begin{array}{l}300 \\
U p \\
210\end{array}$ & $\begin{array}{l}.032 \\
.028 \\
.045\end{array}$ & $\ldots$ & & & 045 & .033 & $\cdots$ \\
\hline \multirow[t]{2}{*}{$210 / 06: 43: 52.54$} & 1.5 & $\begin{array}{l}300 \\
\text { Up }\end{array}$ & $\begin{array}{l}.054 \\
.022\end{array}$ & $\ldots$ & \multicolumn{4}{|c|}{ Keenwild } & \\
\hline & . & & & . & $198 / 20: 35: 20.55$ & 4.0 & $\begin{array}{l}180 \\
\text { Up } \\
090\end{array}$ & $\begin{array}{l}.024 \\
.029 \\
.021\end{array}$ & --- \\
\hline
\end{tabular}


Table 2.--Aftershock accelerograph data for USGS stations in the greater Palm Springs region--Continued

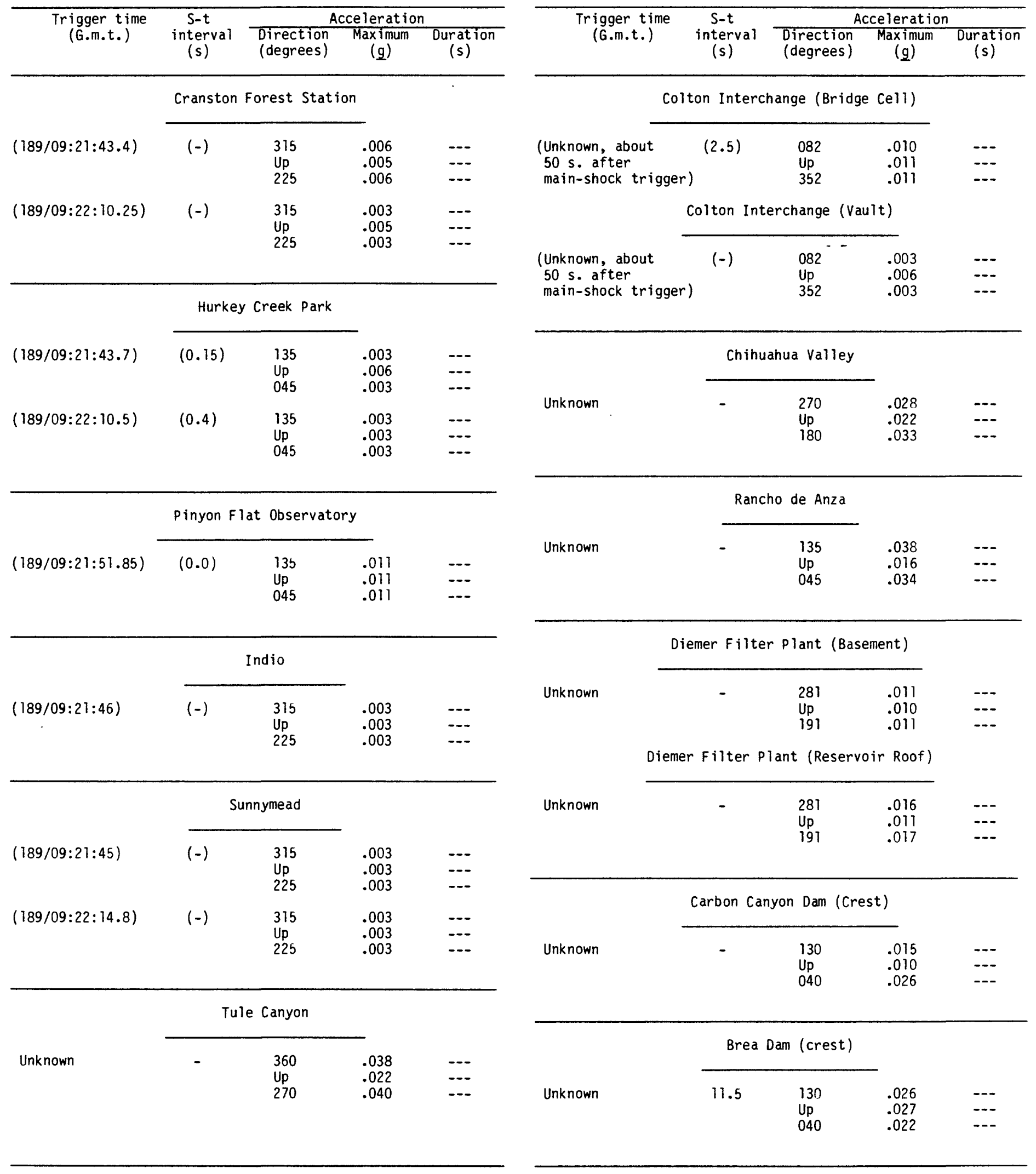


Table 2.--Aftershock accelerograph data for USGS stations in the greater Palm Springs region--Continued

\begin{tabular}{|c|c|c|c|c|c|}
\hline \multirow{2}{*}{\multicolumn{2}{|c|}{$\begin{array}{c}\text { Trigger time } \\
\text { (G.m.t.) }\end{array}$}} & \multirow{2}{*}{$\begin{array}{c}\text { S-t } \\
\text { interval } \\
(s)\end{array}$} & \multicolumn{3}{|c|}{ Acceleration } \\
\hline & & & $\begin{array}{l}\text { Direction } \\
\text { (degrees) }\end{array}$ & $\begin{array}{l}\text { Maximum } \\
(g)\end{array}$ & $\begin{array}{l}\text { Duration } \\
\text { (s) }\end{array}$ \\
\hline \multicolumn{6}{|c|}{ Jensen Filter Plant (Admin. Bldg. Basement) } \\
\hline \multirow[t]{2}{*}{ Unknown } & & - & $\begin{array}{l}025 \\
\text { Up } \\
295\end{array}$ & $\begin{array}{l}.006 \\
.003 \\
.006\end{array}$ & --- \\
\hline & \multicolumn{5}{|c|}{ Jensen Filter Plant (Generator Room Basement) } \\
\hline Unknown & & - & $\begin{array}{l}025 \\
\text { Up } \\
295\end{array}$ & $\begin{array}{l}.005 \\
.003 \\
.006\end{array}$ & --- \\
\hline
\end{tabular}

\begin{tabular}{|c|c|c|c|c|}
\hline \multirow[b]{2}{*}{$190 / 00: 12: 34.2$} & \multicolumn{3}{|c|}{ Hot Springs, Mission Lakes* } & \multirow[b]{2}{*}{$\begin{array}{l}-- \\
-- \\
--\end{array}$} \\
\hline & 1.55 & $\begin{array}{l}360 \\
\text { Up } \\
270\end{array}$ & $\begin{array}{l}.085 \\
.049 \\
.075\end{array}$ & \\
\hline $190 / 09: 41: 23$ & 1.55 & $\begin{array}{l}360 \\
\text { Up } \\
270\end{array}$ & $\begin{array}{l}.037 \\
.025 \\
.023\end{array}$ & 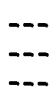 \\
\hline $190 / 20: 10: 36.9$ & 1.55 & $\begin{array}{l}360 \\
\text { Up } \\
270\end{array}$ & $\begin{array}{l}.016 \\
.010 \\
.017\end{array}$ & --. \\
\hline $194 / 01: 41: 41$ & 2.2 & $\begin{array}{l}360 \\
\text { Up } \\
270\end{array}$ & $\begin{array}{l}.016 \\
.020 \\
.017\end{array}$ & $\begin{array}{l}--. \\
-- \\
--\end{array}$ \\
\hline $198 / 20: 35: 18$ & 1.9 & $\begin{array}{l}360 \\
\text { Up } \\
270\end{array}$ & $\begin{array}{l}.048 \\
.044 \\
.133\end{array}$ & $\begin{array}{l}--- \\
--. \\
0.2\end{array}$ \\
\hline $198 / 21: 54: 48$ & 1.8 & $\begin{array}{l}360 \\
\text { Up } \\
270\end{array}$ & $\begin{array}{l}.063 \\
.020 \\
.069\end{array}$ & --- \\
\hline $199 / 19: 58: 04$ & 1.5 & $\begin{array}{l}360 \\
\text { Up } \\
270\end{array}$ & $\begin{array}{l}.011 \\
.010 \\
.012\end{array}$ & $\begin{array}{l}--- \\
--- \\
--\end{array}$ \\
\hline $210 / 06: 43: 52.25$ & 1.6 & $\begin{array}{l}360 \\
\text { Up } \\
270\end{array}$ & $\begin{array}{l}.026 \\
.025 \\
.035\end{array}$ & --- \\
\hline $212 / 07: 51: 44.8$ & 1.6 & $\begin{array}{l}360 \\
\text { Up } \\
270\end{array}$ & $\begin{array}{l}.021 \\
.015 \\
.017\end{array}$ & --- \\
\hline
\end{tabular}

\begin{tabular}{|c|c|c|c|c|}
\hline \multirow{2}{*}{$\begin{array}{c}\text { Trigger time } \\
\text { (G.m.t.) }\end{array}$} & \multirow{2}{*}{$\begin{array}{c}\text { S-t } \\
\text { interval } \\
\text { (s) }\end{array}$} & \multicolumn{3}{|c|}{ Acceleration } \\
\hline & & $\begin{array}{l}\text { Direction } \\
\text { (degrees) }\end{array}$ & $\begin{array}{l}\text { Maximum } \\
(\mathrm{g})\end{array}$ & $\begin{array}{l}\text { Duration } \\
\text { (s) }\end{array}$ \\
\hline \multicolumn{5}{|c|}{ Desert Hot Springs, Mission Lakes--Continued } \\
\hline $241 / 07: 46: 56.6$ & 1.5 & $\begin{array}{l}360 \\
\text { up } \\
270\end{array}$ & $\begin{array}{l}.132 \\
.089 \\
.116\end{array}$ & \begin{tabular}{l}
1 peak \\
\hdashline 0.1
\end{tabular} \\
\hline $252 / 16: 22: 52.65$ & 1.5 & $\begin{array}{l}360 \\
\text { Up } \\
270\end{array}$ & $\begin{array}{l}.026 \\
.044 \\
.029\end{array}$ & $\begin{array}{l}--- \\
-\cdots \\
---\end{array}$ \\
\hline $271 / 07: 06: 31.7$ & - & $\begin{array}{l}360 \\
\text { up } \\
270\end{array}$ & $\begin{array}{l}.021 \\
.010 \\
.012\end{array}$ & $\begin{array}{l}--- \\
---\end{array}$ \\
\hline $288 / 02: 28: 50.0$ & 1.5 & $\begin{array}{l}360 \\
\text { Up } \\
270\end{array}$ & $\begin{array}{l}.106 \\
.049 \\
.087\end{array}$ & $\begin{array}{l}0.15 \\
--- \\
---\end{array}$ \\
\hline
\end{tabular}

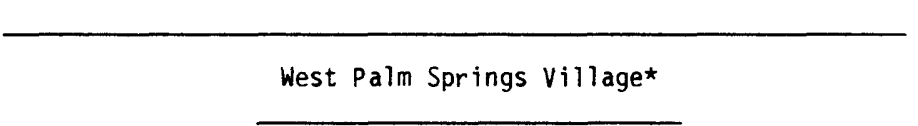

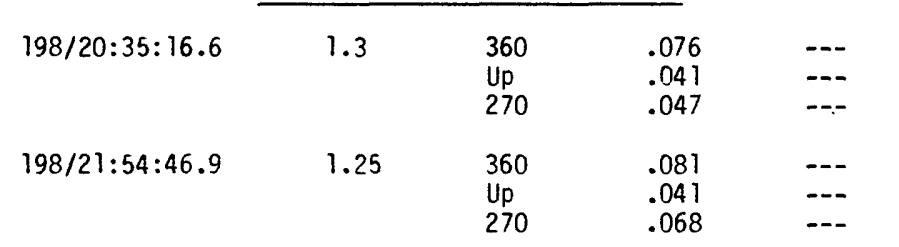

\begin{tabular}{|c|c|c|c|c|}
\hline & \multicolumn{2}{|c|}{ Mill ard Canyon* } & \multirow[b]{2}{*}{$\begin{array}{l}.019 \\
.011 \\
.017\end{array}$} & \multirow[b]{2}{*}{$\begin{array}{l}--- \\
---\end{array}$} \\
\hline $198 / 21: 54: 50.6$ & - & $\begin{array}{l}360 \\
\text { Up } \\
270\end{array}$ & & \\
\hline Unknown & - & $\begin{array}{l}360 \\
\text { Up } \\
270\end{array}$ & $\begin{array}{l}.006 \\
.003 \\
.006\end{array}$ & $\begin{array}{l}-- \\
--- \\
--\end{array}$ \\
\hline
\end{tabular}

\begin{tabular}{|c|c|c|c|c|}
\hline \multirow[b]{2}{*}{$198 / 20: 35: 17.27$} & \multicolumn{3}{|c|}{ Canyon House, Morongo Valley* } & \\
\hline & - & $\begin{array}{l}360 \\
\text { Up } \\
270\end{array}$ & $\begin{array}{l}.079 \\
.072 \\
.082\end{array}$ & $\begin{array}{l}--- \\
--- \\
--\end{array}$ \\
\hline
\end{tabular}

*Temporary aftershock station. 